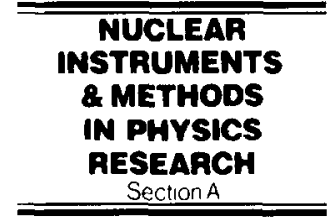

\title{
An update on the NIST radon-in-water standard generator: its performance efficacy and long-term stability
}

\author{
R. Collé*, Raj Kishore ${ }^{1}$ \\ Physics Laboratory, National Institute of Standards and Technology, Gaithershurg, MD 20899, USA
}

Received 23 July 1996; revised form received 3 February 1997

\begin{abstract}
A ${ }^{226} \mathrm{Ra}-{ }^{222} \mathrm{Rn}$ generator that could he used as a transfer standard for radon-in-water measurement calibrations was previously developed and described. The generator utilized a novel ${ }^{222} \mathrm{Rn}$ emanation source that was comprised of a ${ }^{226} \mathrm{Ra}$ solution encapsulated in polyethylene. The long-term performance of this standard generator has now been investigated and evaluated. The evaluation included exhaustive and more reliable measurement uncertainty analyses for the generator's performance and routine operation. Modifications to the original protocol for operation of the generator has also resulted in improved precision in the ${ }^{222} \mathrm{Rn}$ activity concentration in a dispensed aliquant. The evaluation results indicate that the generator performance has remained stable. and that the calibration parameters are still well within their given uncertainty intervals for the originally determined canonical values. Over a period of approximately six years, experimentally determined values of the ${ }^{222} \mathrm{Rn}$ emanation fraction, the most critical parameter, have remained constant and invariant of conditions within statistical variations of about $0.3 \%$ (corresponding to a relative standard deviation of the mean). All evaluation measurements were performed by $4 \pi-\alpha \beta$ liquid scintillation spectrometry of gravimetrically determined aliquants dispensed from the generator.
\end{abstract}

Keywords: Calibration; Emanation; Liquid scintillation; Measurement; Polyethylene; Radioactivity; Radium-226; Radon-222; Standard: Water

\section{Introduction}

In 1986, the National Institute of Standards and Technology (NIST, then called the National Bureau of Standards) completed the development of a "generator" that could be used as a transfer standard for ${ }^{222} \mathrm{Rn}$-in-water measurement calibrations $[1,2]$. The standard is primarily intended for use with liquid scintillation (LS) measurements of aqueous ${ }^{222} \mathrm{Rn}$ concentrations [3-9]. The generator ${ }^{2}$ consists of a polyethylene-encapsulated

\footnotetext{
* Corresponding author.

1 NIST guest research scientist (1989-1991): presently at Otsuka American Pharmaceutical, Inc., Rockville, MD USA.

2 An earlier prototype of the generator [1] utilized a solid source of ${ }^{226} \mathrm{Ra}$ which was deposited on an ion-exchange filter and sandwiched between two layers of thin polyethylene tape. Although this solid-source generator was subsequently found to be inadequate in terms of its stability over long times, this earlier work established the validity of the polyethylene-diffusion principle as applied to the encapsulated ${ }^{226} \mathrm{Ra}$ sources, and it gave other physical characteristics of the generator system.
}

${ }^{226} \mathrm{Ra}$ solution source in a small-volume accumulation
chamber and an ancillary mixing and dispensing system
which is partially automated with motor-driven, $50 \mathrm{ml}$
"gas-tight" syringes. The entire, self-contained generator
is maintained and operated under air-free conditions.
Unlike other ${ }^{22} \mathrm{Rn}$-in-water calibration standards then
and still in use (cf. Refs [5-9]), this standard can be used
to generate and accurately dispense radium-free ${ }^{222} \mathrm{Rn}$
solutions of known concentration, without invoking as-
sumptions or difficult corrections for a ${ }^{226} \mathrm{Ra}$ component
in the calibration standard. Use of ${ }^{226} \mathrm{Ra}$ calibration
standards (sometimes with large accompanying ${ }^{210} \mathrm{~Pb}$
concentrations that are poorly quantified [10]) for LS
measurements of ${ }^{222} \mathrm{Rn}$ can introduce significant analyti-
cal uncertainties. Many, if not all (cf. Refs. [6-9]),
so-called ${ }^{22.2} \mathrm{Rn}$ measurement "calibration protocols"
that rely on ${ }^{226} \mathrm{Ra}$ sources. such as those utilizing
immiscible mineral-oil/water LS cocktails, will not
withstand critical scrutiny. These calibration standards,
using either ${ }^{226} \mathrm{Ra}$ on selective ion-exchange resins
or ${ }^{226} \mathrm{Ra}$ solutions directly, rely upon the presump-
tion that the ${ }^{226} \mathrm{Ra}$ (and possibly ${ }^{210} \mathrm{~Pb}$ ) remains 
exclusively in the aqueous phase without an LS counting interference.

The standard generator, as described previously $[1,2]$, was calibrated and certified in terms of several parameters (most importantly the ${ }^{222} \mathrm{Rn}$ emanation fraction $f$ ) that allow calculation of the ${ }^{222} \mathrm{Rn}$ concentration or total activity in an aliquant dispensed from the generator when a detailed operating protocol is rigorously followed. The total propagated uncertainty of the calibration, in terms of an assumed relative standard deviation, was estimated at the time to be approximately $1.2 \%$. The original standard generator, as described by Hutchinson et al. [2], was delivered and reposited at the Las Vegas laboratory of the U.S. Environmental Protection Agency (EPA) in 1987. Readers may wish to consult the original references $[1,2]$ for detailed schematics that illustrate the construction and operation of the generator.

In 1988, NIST initiated the fabrication of a nearly identical, duplicate generator. It was intended for internal use at NIST, and for evaluations of the performance of the generator over time. Representative examples of its internal use at NIST include a verification of a bias in the calibration of electret-based integral radon monitors [11] and preparation of samples used for a precise determination of the ${ }^{222} \mathrm{Rn}$ half-life [12]. A version of this generator, incorporating identical operating principles and a similar polyethylene-encapsulated ${ }^{226} \mathrm{R}$ a source, is also reported to be under development for use as a national calibration standard in UK [13].

Since the publication of the earlier papers $[1,2]$, the generator has undergone various evaluations of its longterm stability and performance efficacy. This paper describes the results of these evaluations, and provides more reliable uncertainty estimates. Modifications to the original operating protocol for the generator [2], resulting in improved precision in the ${ }^{22}{ }^{2} \mathrm{Rn}$ activity concentration for a dispensed aliquant, are also given.

\section{Generator and experimental considerations}

The source used for this second standard generator consists of a heat-sealed polyethylene capsule having a total mass of approximately $288 \mathrm{mg}$, and containing (initially) approximately $170 \mathrm{mg}$ of a ${ }^{226} \mathrm{Ra}$ solution in $118 \mathrm{mg}$ of low-density polyethylene. The solution consists of a calibrated ${ }^{226} \mathrm{Ra}$ solution of nominal $1 \mathrm{moll}^{-1}$ hydrochloric acid containing approximately $2 \mathrm{mg} \mathrm{BaCl} 2$ per gram of solution. The capsule is in a right-circular cylinder configuration with an outer diameter of roughly $0.4 \mathrm{~cm}$, a length of about $2.0 \mathrm{~cm}$, and a nominal average wall thickness of $0.07 \mathrm{~cm} .{ }^{3}$ It contains a total ${ }^{226} \mathrm{Ra}$ activity of $1167 \pm 11 \mathrm{~Bq}$ as of 9 September 1991, where the cited uncertainty is an "expanded uncertainty". At the time of the initial encapsulation of the solution, the capsule contained a small air void estimated to bc less than $0.008 \mathrm{~cm}^{3}$ out of a total internal volume of about $0.180 \mathrm{~cm}^{3}$. The capsule was subsequently kept completely immersed in water within the generator's accumulation chamber. Over the passage of several years, the air void diminished as a result of transpiration of water into the capsule until the void was no longer visible. The ratio of total polyethylene mass to total water mass in the accumulation chamber was more favorable in this second generator (in terms of ${ }^{222} \mathrm{Rn}$ emanation) compared to the earlier generator located at EPA. ${ }^{5}$ All other fabrication details for the generator, such as the materials used (gas-tight syringes, valves, joints. and tubings, etc.) and

3 The capsule before filling was fabricated by hand-crafted drawing of a polyethylene tube and therefore is not likely to have a constant wall thickness across its length. The equivalent average wall thickness was estimated from the total and polyethylene masses, known densities, and considerations of the geometry and exterior dimensions.

${ }^{4}$ The specification of measurement uncertainties and the uncertainty analysis procedures used throughout this paper follow the normal conventions of the NIST Radioactivity Group. These conventions are wholly compatible with those adopted by the principal international metrology standardization bodies $[9,10]$. All individual uncertainty components are expressed in terms of estimated (experimental) standard deviations (or standard deviations of the mean where appropriate) or quantities assumed to correspond to standard deviations irrespective of the method used to evaluate their magnitude. All of these component quantities are designated as "standard uncertainties." A propagated uncertainty, termed a "combined standard uncertainty," is expressed as what is assumed to be an estimated standard deviation which is equal to the positive square root of the total variance obtained by summing all variance (square of the standard uncertainty) and covariance components, however evaluated. using the law of propagation of uncertainty for the specific mathematical function given by the model of the measurement procedure [9]. By recently established NIST policy [10]. the combined standard uncertainty is multiplied by a "coverage factor" of $k=2$ to obtain an "expanded uncertainty" which is assumed to provide an uncertainty interval with a confidence of roughly $90-95 \%$. For comparative purposes. it should be noted that previous certifications (e.g.., for Standard Reference Materials) issued by the NIST Radioactivity Group used comparably-based uncertainty coverage factors of $k=3$. This former practice was historically rooted and was assumed to provide certified uncertainty intervals with somewhat higher confidence levels, approaching 95-99\%.

5 The capsule dimensions and polyethylene and solution masses reported for this earlier generator, as given by Hutchinson, et al. [2] are wholly inconsistent. We strongly suspect that the reported polyethylene mass given therein is in error by perhaps as much as a factor of two or more. Excepting the careful gravimetric filling of the earlier capsule with the ${ }^{226} \mathrm{Ra}$ solution, neither the constituent masses nor physical dimensions were as controlled (or as documented) as for the preparation of the source used in the present generator described here. 
internal volumes, were virtually identical to those described earlier [2].

Operating details were also nearly identical to those given earlier [2]. The principal differences were improvements in determining the total dilution of the accumulated ${ }^{222} \mathrm{Rn}$ activity and the dispensed aliquant sizes, and in reproducing the total filling volume.

Originally, the standard generator was calibrated and certified on a volume basis in terms of the ${ }^{222} \mathrm{Rn}$ activity concentration $K$ or total activity $A$ in a given aliquant of volume $v$ dispensed from the generator. These quantities could be calculated from a general expression containing certified parameters, when the accompanying operating protocol was invoked. The following parameters were certified: the total ${ }^{226} \mathrm{Ra}$ activity in the encapsulated source $A_{0}$; the ${ }^{222} \mathrm{Rn}$ emanation fraction $f$; a "dead volume" (given by the volume of tubing between the two syringes) which was used along with the filling volume of the two syringes to obtain the total dilution volume $V$; and, for convenience, a conversion factor $k_{\mathrm{x}}$ for the volume of a dispensed aliquant $v$ in terms of the number of turns on the dispensing-syringe motordrive. ${ }^{6}$ Since then, it was found that substantially greater precision could be achieved by firstly increasing the "dead volume" to reproduce its magnitude more precisely, and secondly by directly measuring the total mass of blank water $m_{\mathrm{D}}$ used to perform the dilution. The revised procedure tries to ensure that the starting position of the syringes (which defines the "dead volume") is returned to, as nearly as possible, at the end of the dispensing operations. This was achieved by slightly off-setting the syringe plunger from a fully inserted position and thereby increasing $m_{\mathrm{d}}$. The total dilution mass $M$ can be given by the mass of this "dead volume" $m_{d}$ plus the mass $m_{D}$. The dilution mass $m_{\mathrm{D}}$ is determined gravimetrically by adding the masses $m$ of all component aliquants dispensed from the generator plus the initial mass of solution used to flush the dispensing needle and the mass of any remaining solution in the dispensing syringe. In other words, the total mass of all of the solution that exits the generator in returning the syringes to their starting positions is directly measured. The "dead volume" $m_{\mathrm{d}}$ is estimated from dimensional measurements and geometrical considerations. It must be noted however, that the emanation fraction $f$, as defined and cxpcrimentally determined, is a function of $m_{\mathrm{d}}$. The total [or "absolute" (sic)] uncertainty in determining $m_{\mathbf{d}}$ is therefore unimportant. Rather, it is only the uncertainty in reproducing $m_{d}$ from one use of the generator to the next that propagates as an uncertainty component for the accumulated and diluted ${ }^{222} \mathrm{Rn}$ activity concentration. This uncertainty component, in turn, obviously, is merely dependent on the

\footnotetext{
${ }^{6}$ The dispensing conversion factor was actually determined in terms of the mass of dispensed aliquant per turn [2].
}

reproducibility in returning the syringes to their starting positions.

Following the notation previously utilized [2], the ${ }^{222} \mathrm{Rn}$ activity concentration $K$ and the total ${ }^{222} \mathrm{Rn}$ activity $A$ in an aliquant dispensed from the generator are thus given by

$K=A / m=f A_{0} \exp \left(-\lambda_{\mathrm{Ra}} t_{\mathrm{d}}\right)\left[1-\exp \left(-\lambda_{\mathrm{Rn}_{\mathrm{n}}} t_{\mathrm{a}}\right)\right] / M$,

where $m$ is the gravimetrically measured mass of a dispensed aliquant; $f$ is the source emanation fraction (the fraction of the total ${ }^{222} \mathrm{Rn}$ generated in the source by the decay of ${ }^{226} \mathrm{Ra}$ that is released to the water in the accumulation chamber); $A_{0}$ is the total ${ }^{226} \mathrm{Ra}$ activity in the source at reference time $t_{0} ; \hat{\lambda}_{\mathrm{Ra}}$ is the ${ }^{226} \mathrm{Ra}$ decay constant; $t_{\mathrm{d}}$ is a decay time interval given by the difference between $t_{0}$ and the start time $t_{1}$ of an accumulation; $\lambda_{R_{n}}$ is the ${ }^{222} \mathrm{Rn}$ decay constant; $t_{\mathrm{a}}$ is an accumulation time interval given by difference between $t_{1}$ and the end time $t_{2}$ of an accumulation; and $M$ is the total mass of solution containing ${ }^{222} \mathrm{Rn}$ which is given by a defined "dead volume" $m_{\mathrm{d}}$ and a gravimetrically-measured dilution mass $m_{\mathrm{D}}$. The adopted decay constants are $\lambda_{\mathrm{Ra}}=$ $(1.186 \pm 0.005) 10^{-6} \mathrm{~d}^{-1} \quad[16]$ and $\lambda_{R \pi}=0.18130 \pm$ $0.00002 \mathrm{~d}^{-1}[17]$.

For routine operation of the generator, the available ${ }^{222} \mathrm{Rn}$ activity concentration $K$ may be varied by adjusting the accumulation time $t_{\mathrm{a}}$ and the total solution mass $M$. For $M \simeq 55 \mathrm{~g}, K$ ranges from about $2 \mathrm{~Bq} \mathrm{~g}^{-1}$ for an accumulation with $t_{\mathrm{a}} \simeq 1 \mathrm{~d}$ to about $14 \mathrm{Bqg}^{-1}$ for $t_{\mathrm{a}}>20 \mathrm{~d}$. This range may be increased to higher concentrations by use of smaller dilution volumes (and $M$ ). Operation of the generator, however, has been found to be most reproducible by using a near maximum $M \sim 55 \mathrm{~g}$.

All of the performance evaluation measurements described herein were based on $4 \pi-\alpha \beta$ liquid scintillation (LS) spectrometry of gravimetrically determined aliquants dispensed from the generator. The routine gravimetric procedures used in our laboratory as they apply to the preparation of LS counting sources, as well as estimations of their associated uncertainties, have been treated at length previously [18-22]. Mass determinations for the dispensed aliquants $m$ have relative standard uncertainties of about $\pm 0.05 \%$. The generalur dispensings and LS measurement procedures used for this study were similar to those given earlier [2]. Two different LS spectrometers were employed. Their principal operating characteristics are outlined in Table 1. Descriptions of these operating characteristics, their respective performance, and use within this laboratory for various radionuclidic calibrations are available [18-22]. Specific aspects of the LS procedures as they apply to ${ }^{222} \mathrm{Rn}$ measurements have been given by Collé, et al. $[11,12]$. The LS counting sources (i.e., cocktails) contained ${ }^{222} \mathrm{Rn}$-laden aqueous solutions ranging from as small as 
Table 1

Characteristics of the LS spectrometers used for the evaluations of the Radon-In-Water Standard Generator

\begin{tabular}{|c|c|c|}
\hline Characteristic & System B & System P \\
\hline LS spectrometer model & Beckman LS7800 & Packard Tri-carb A2500TR \\
\hline Operating mode & Sum-coincidence & Sum-coincidence \\
\hline Photomultiplier tubes & Hamamatsu R331-05 & Hamamatsu R331-08 \\
\hline Operating temperature & Ambient & Ambient \\
\hline Coincidence resolving time & $22 \mathrm{~ns}$ & $18 \mathrm{~ns}$ \\
\hline Sum-coincident pulse amplification & Logarithmic & Linear \\
\hline Pulse resolving time & $\begin{array}{l}5-33 \mu \mathrm{s} \\
\text { (variable with pulse height) }\end{array}$ & $\begin{array}{l}12 \mu \mathrm{s} \\
\text { (fixed) }\end{array}$ \\
\hline $\begin{array}{l}\text { Spectral analog-to-digital converter } \\
\text { (ADC) capacity }\end{array}$ & 1000 channels & 2048 channels \\
\hline $\begin{array}{l}\text { Nominal conversion gain } \\
\text { (energy per channel) }\end{array}$ & $\begin{array}{l}\text { Variable } \\
\text { (with logarithmic energy) }\end{array}$ & $\simeq 1 \mathrm{keV}$ \\
\hline Detection threshold (nominal) & $\leq 1 \mathrm{keV}$ & $\leq 1 \mathrm{keV}$ \\
\hline $\begin{array}{l}\text { Live-time determination method } \\
\text { (and uncertainty) }\end{array}$ & $\begin{array}{l}\text { Gated oscillator (scaled) } \\
( \pm 0.1 \%)\end{array}$ & $\begin{array}{l}\text { Gated oscillator (scaled) } \\
( \pm 0.1 \%)\end{array}$ \\
\hline Quench indicating parameter (QIP) & Horrocks number $(H)$ & $\begin{array}{l}\text { Transformed Spectral Index of the } \\
\text { External Standard (tSIE) (proprietary) }\end{array}$ \\
\hline $\begin{array}{l}\text { External } \gamma \text {-ray source for } \\
\text { QIP determination }\end{array}$ & ${ }^{137} \mathrm{Cs}$ & ${ }^{1,3} \mathrm{Ba}$ \\
\hline (and location) & (sidc) & (bottom) \\
\hline
\end{tabular}

Table 2

Scintillants (commercially prepared) used for the evaluations of the Radon-In-Water Standard Generator

\begin{tabular}{lllll}
$\begin{array}{l}\text { Commercial } \\
\text { scintijlant }\end{array}$ & $\begin{array}{l}\text { Acronym } \\
\text { descriptor }\end{array}$ & Manufacturer & $\begin{array}{l}\text { Density } \\
\left(\mathrm{g} \mathrm{mll}^{-1}\right)\end{array}$ & Composition \\
\hline $\begin{array}{llll}\text { PCS } \\
\text { Ready Safe }\end{array}$ & PCS & Amersham & 0.92 & $\begin{array}{l}\text { Xylene; 2-ethoxyethanol (a surfactant); unspecified fluor } \\
\text { Phenylxylylethane (PXE), 50-80\%; non-ionic surfactant, 20-50\%; }\end{array}$ \\
Instagel XF & IG & Peckman & 0.97 & $\begin{array}{l}2,5 \text {-diphenyloxazole (PPO). < } \% \% \\
1,2,4 \text {-trimethylbenzene (pseudocumene); with unspecified emulsi- } \\
\text { fiers; PPO and bis(2-methylstyryl)benzene (bis-MSB) fluors }\end{array}$ \\
\hline
\end{tabular}

a few tenths of a gram up to nearly $5 \mathrm{~g}$ in various proportions (typically $15-20 \mathrm{ml}$ ) of several commercially available scintillants (Table 2 ). ${ }^{7}$ The cocktails were contained in glass, nominal $20 \mathrm{ml}$ LS vials having aluminum-backed liners on plastic screw caps. Previous studies to evaluate possible radon losses in the LS vials, and to determine ${ }^{222} \mathrm{Rn}$ LS detection efficiencies, were made in flame-sealed glass LS vials [12]. In all cases, the LS cocktails were compared with matched blanks of nearly identical composition for background subtractions. The blanks were prepared with aged, ion-exchanged, "radon-

7 Most initial measurements in the period prior to 1991 exclusively used the xylene-based PCS scintillant, which is no longer commercially available. The other scintillants (RS and IG) were intereompared to PCS in an interim period from 1991 to 1994. Only the latter two scintillants were used since 1994. free", doubly distilled water having a total $\alpha$ - or $\beta$-emitting radionuclidic impurity, in terms of an equivalent ${ }^{222} \mathrm{Rn}$ activity concentration, of less than $0.003 \pm 0.001$ $\mathrm{Bqg}^{-1}$. Generator dilutions were performed with the same blank water supply. Measurements were initiated only after the cocktails contained ${ }^{222} \mathrm{Rn}$ in radioactive cquilibrium with its short-lived ${ }^{218} \mathrm{Po}^{214} \mathrm{~Pb}^{214} \mathrm{Bi}-$ ${ }^{214}$ Po subseries (i.e., after a minimum of 5-6h). The overall LS detection efficiency is almost exactly 5 counts $\mathrm{s}^{-1} \mathrm{~Bq}^{-1}$ resulting from a very nearly $100 \%$ efficiency for the three $\alpha$ decays $\left({ }^{222} \mathrm{Rn},{ }^{218} \mathrm{Po}\right.$, and ${ }^{214} \mathrm{Po}$ ) and an almost comparable $100 \%$ efficiency for the two energetic $\beta$ decays $\left({ }^{214} \mathrm{~Pb}\right.$ and $\left.{ }^{214} \mathrm{Bi}\right)$. The efficiency is nearly invariant of reasonable sample quenching, but is dependent on the water fraction in the cocktail (as it affects the radon solubility) and on the size of the air space in the LS vial above the cocktail. Based on an efficiency tracing method [23-25] using theoretical 


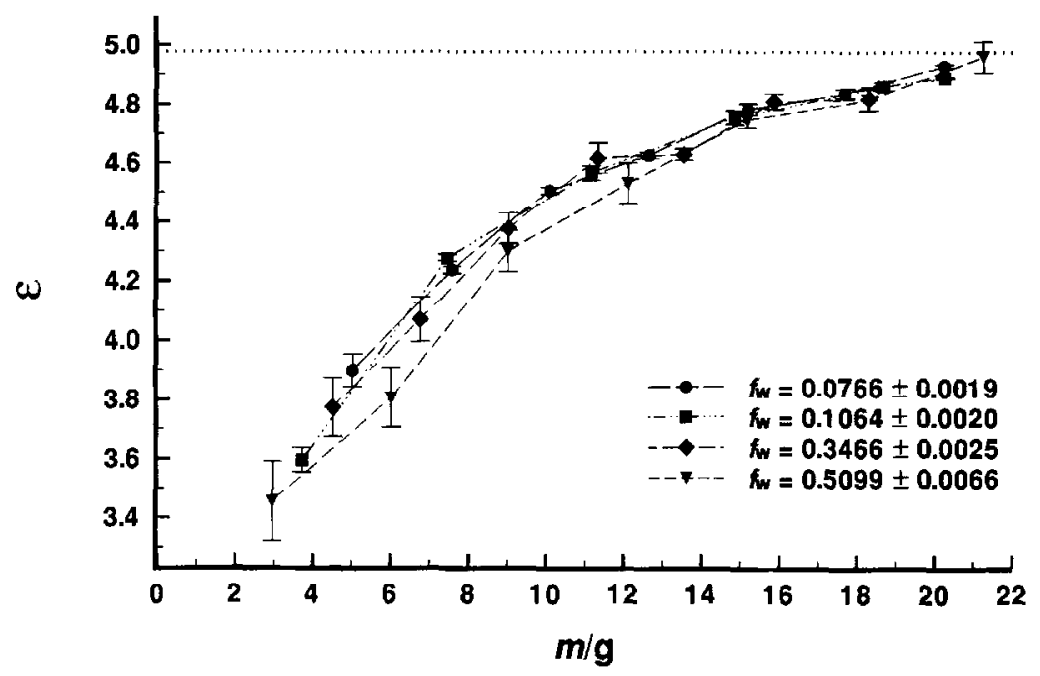

Fig. 1. Experimentally determined LS detection efficiencies $\varepsilon$ for ${ }^{222} \mathrm{Rn}$ subseries decay as a function of total cocktail mass $m_{c}$ (in units of g) for four $\mathrm{H}_{2} \mathrm{O}$ mass fractions $f_{\mathrm{w}}$. Each cocktail consists of $f_{\mathrm{w}} m_{\mathrm{v}} \mathrm{g}$ of ${ }^{222} \mathrm{Rn}$-laden $\mathrm{H}_{2} \mathrm{O}$ in $\left(1-f_{\mathrm{w}}\right) m_{\mathrm{c}} \mathrm{g}$ of PCS scintillant in a nominal, flame-sealed $20 \mathrm{ml}$ glass LS vial. The value of $\varepsilon_{s}$ (cited in the text) for an "unquenched" cocktail with no radon losses to the air space is shown by the dotted line. The uncertainty interval on each datum correspond to $v_{\mathrm{c}}$ for $6 \leq n_{\mathrm{m}} \leq 12$ replicate measurements on each cocktail (see text).

modelling calculations and measurements with matched sets of ${ }^{3} \mathrm{H}$-standard LS cocktails, the LS counting efficiency for the ${ }^{222} \mathrm{Rn}$ subseries in "unquenched" (sic) samples was found to be $\varepsilon_{\mathrm{s}}=4.977_{-0.015}^{+0} \mathrm{nng}$ counts $\mathrm{s}^{-1} \mathrm{~Bq}^{-1}$ [12]. The efficiency-tracing quench corrections included appropriate extrapolations for the scintillant-to-water ratio in the LS cocktail; for partitioning of radon between the scintillant-water cocktail mix and the air space in the LS vials; and for count-rate-versus-energy extrapolations to zero energy. Fig. 1 illustrates some representative experimental efficiencies for the ${ }^{222} \mathrm{Rn}$ subseries as functions of the cocktail compositions variables: $f_{\mathrm{w}}$, the $\mathrm{H}_{2} \mathrm{O}$ mass fraction in the cocktail; and $m_{\mathrm{e}}$, the total cocktail mass. ${ }^{3}$ LS cocktail stability over the measurement times was typically estimated from variations in quench indicating parameters [20]. The ${ }^{222} \mathrm{Rn}$ LS detection efficiency was independently verified [2] by confirmatory measurements with the NIST pulse-ionization-chamber-based primary ${ }^{222} \mathrm{Rn}$ measurement system which serves as the U.S. national radon measurement standard [26, 27].

The generator, for any given performance evaluation, was used to dispense and prepare a series of $n_{\mathrm{s}} \mathrm{LS}$ samples, ranging from $n_{\mathrm{s}}=6$ to $n_{\mathrm{s}}>20$. The evaluations used varying, but well-determined, accumulation times $t_{\mathrm{a}}$ from $t_{\mathrm{a}} \simeq 0.9 \mathrm{~d}$ to $t_{\mathrm{a}}>40 \mathrm{~d}$. The relative standard uncertainty in $t_{\mathrm{a}}$ (due largely to the finite time needed to dilute and transfer the ${ }^{222} \mathrm{Rn}$ in the accumulation cham-

\footnotetext{
${ }^{8}$ For a cocktail with given $f_{w}$ and $m_{\mathrm{c}}$, the cocktail volume $v_{\mathrm{e}}$ may be approximated by $t_{\mathrm{c}} \simeq m_{\mathrm{c}}\left[f_{\mathrm{w}}+\left(1-f_{\mathrm{w}}\right) / \rho\right]$ where $p$ is the density of the scintillant (Table 2).
}

ber) ranged from approximately $0.05 \%$ for a $t_{\mathrm{a}} \simeq 1 \mathrm{~d}$ accumulation to about $0.002 \%$ for a $t_{\mathrm{a}} \simeq 30 \mathrm{~d}$ accumulation. Each of the dispensed ${ }^{222} \mathrm{Rn}$ solution aliquants used to prepare the LS cocktails had an independently determined mass $m_{j}$ (with $j=1,2,3, \ldots, n_{\mathrm{s}}$ ). Each sample, along with matched blanks for background subtractions, was then measured on either LS spectrometer a number $n_{\mathrm{m}}$ replicate times, which was typically in the range $5 \leq n_{\mathrm{m}} \leq 12$ but which varied (for special tests) from a minimum of $n_{\mathrm{m}}=3$ to $n_{\mathrm{m}}=120$. The counting time intervals for each LS measurement was typically 15 or $20 \mathrm{~min}$ in duration, and ranged from 5-60 $\mathrm{min}$ in extremes. The decay-corrected, net counting rate concentration for sample $j$ during measurement cycle $\mathbf{i}$ (with $i=1,2,3, \ldots, n_{\mathrm{m}}$ ) was obtained from

$R_{j, i}=\left[C_{j, i}-C_{B(i)}\right] /\left[\exp \left(-\hat{\lambda}_{\mathrm{Rn}} T_{i}\right) t_{i} m_{j}\right]$.

where $C_{j . i}$ is the gross integral counts in the full-energy LS spectrum for the $j$ th sample at measurement time $T_{i}$ : $C_{\mathrm{B}(i)}$ is the corresponding gross integral counts for background obtained from a spectrum of a matched blank LS sample in the same $i$ th counting cycle; $\lambda_{\mathrm{R}_{\mathrm{n}}}$ is the ${ }^{222} \mathrm{Rn}$ decay constant: $T_{i}$ is the decay time interval from the midpoint of the measurement time interval to a common reference time rtypically taken as the previously-defined end time $t_{2}$ of the accumulation); $t_{i}$ is the counting (live) time interval for measurement of both the source and matched blank; and $m_{j}$ is the mass of the dispensed ${ }^{222} \mathrm{Rn}$ solution used in the $j$ th sample.

Routine evaluations of the generator perfornance (over time) were typically performed with $m_{j} \simeq 1.5 \mathrm{~g}$ in 
about $18.5 \mathrm{~g}$ of scintillant to form cocktails with $f_{\mathrm{w}} \simeq 0.1$ and $m_{\mathrm{c}} \simeq 20 \mathrm{~g}$, although other conditions were also employed for special tests as noted below.

\section{Uncertainty (statistical) model considerations}

Generator performance was analyzed and could be better understood by considering the underlying uncertainty components, using one-way-classification analysis of variance (ANOVA) techniques for a random-effects statistical model [28].

Relative standard deviations $v_{P(i)}$ in percent for the total Poisson "counting error" (sic) for any single measurement of $R_{j, i}$ would normally be obtained from the usual expression $100\left[C_{j, i}+C_{B(i)}\right]^{1 / 2} /\left[C_{j, i}-C_{B(i)}\right]$ on assuming that the measurement process is Poisson distributcd. ${ }^{9}$ However, the $t_{i}$ counting intervals used in this study were typically long or comparable to the half-lifes of the individual members of the ${ }^{222} \mathrm{Rn}$ subseries. As a result, the above expression used to estimate the socalled statistical counting error is not valid since the short-lived ${ }^{222} \mathrm{Rn}$ daughters do not follow the Poisson statistics for independently decaying species. Following the methodology of Lucas and Woodward [29] in which the standard deviation associated with the "counting statistics" for long decay chains is given by $s \simeq(J N)^{1 / 2}$ with $J=\sigma^{2} / \mu$ (the quotient of the variance in the mean number of observed events and the mean), the previous expression can he modified to give $v_{P(i)}=100\left[J_{j, i} C_{j, i}\right.$ $\left.+C_{B(i)}\right]^{1 / 2} /\left[C_{j, i}-C_{B(i)}\right]$. Lucas and Woodward [29] have tabulated values of $J$ for the ${ }^{222} \mathrm{Rn}$ subseries for a variety of combinations of counting time interval and detection efficiency under the condition that only the three $\alpha$ emitters in the decay subchain are counted. The LS results presented here detect (in $4 \pi$ geometry) the three $\alpha$ emitters as well as the two $\beta$ emitters with virtually $100 \%$ efficiency so that the tabulations of Lucas and Woodward [29] could not be directly applied. Instead, $J_{j, i}$ factor approximations for each ith measurement and $j$ th sample condition were calculated (using the identical approach of Lucas and Woodward [29]) to estimate $v_{P(i)}$. The exact magnitudes of $v_{P(i)}$, of course, were explicitly dependent on the $t_{i}$ counting times and on the total ${ }^{222} \mathrm{Rn}$ content in the cock tails. The latter in turn was merely dependent on the previously-defined $t_{\mathrm{a}}$ accumulation times and on the $m_{j}$ sample sizes. It may be of

\footnotetext{
9 The term "counting error", a gross misnomer, arises from the assumption that the radioactive decay process itself (but not necessarily the counting process) is Poisson distributed (with equal mean $\mu$ and variance $\sigma^{2}$ for the distribution) such that its variance $\left(N=\sigma^{2}\right)$ is equal to the mean number of total observed counts $(N=\mu)$.
}

interest to note that the first (and usual) expression for the counting errors would result in underestimates by a factor of about 2.2 in the limit of very long counting times - as compared to more-exact calculations of $v_{P(i)}$ using $J$ factors. For the present experimental conditions, the $v_{P(i)}$ would have, in any worse case, been underestimated by a factor of considerably less than 2 and in general would have resulted in underestimates of about $50 \%$ to $60 \%$.

For $n_{\mathrm{m}}$ replicate measurements, the overall relative "counting error" for a single $j$ th sample can be obtained from summing the total counts over all measurements of that sample;

$v_{P}=100\left\{\Sigma_{i}\left[J_{j, i} C_{j, i}+C_{B(i)}\right]^{1 / 2} / \Sigma_{i}\left[C_{j, i}-C_{B(i)}\right]\right\}$.

The "counting error" (assuming no ${ }^{222} \mathrm{Rn}$ decay over the course of the measurements) would reduce to approximately $v_{P} \simeq v_{P(i)} / \sqrt{ } n_{\mathrm{m}}$. For most of the calibration results given herein, values of $v_{p}$ were typically $\leq 0.2 \%$.

The variability in the $R_{j, i}$ values (for constant $j$ ) would reflect the overall LS measurement precision, and would include the $v_{p}$ "counting error" component, as well as any other components of random variation in the measurement process. This measurement variability may be characterized either by a relative standard deviation $v_{\mathrm{C}}$ computed from the data set of $R_{j, i}$ values, or by a relative standard deviation of the mean $v_{C} / \sqrt{ } n_{\mathrm{m}}$ (with $n_{\mathrm{m}}-1$ degrees of freedom). For any given generator run, the $R_{j, i}$ values could be averaged across the $n_{\mathrm{m}}$ measurement cycles to obtain mean $\left\langle R_{j}\right\rangle$ values for each sample in the series. The variability among these $\left\langle R_{j}\right\rangle$ mean values (for constant $m_{j}$ ), which again may be expressed as either calculated relative standard deviations $v_{R}$ or relative standard deviations of the mean $v_{R} / \sqrt{ } n_{\mathrm{s}}$, would reflect the measurement precision given by the $v_{\mathrm{C}} \mathrm{com}$ ponent plus any sample variability. Further averaging the $\left\langle R_{j}\right\rangle$ means across all $n_{\mathrm{s}}$ samples (assuming constant $m_{j}$ ) results in a grand mean $\langle R \gg$ for the given generator run. The accumulated and diluted ${ }^{222} \mathrm{Rn}$ activity concentration in the generator with a mean $\langle K\rangle$ can then be related to $\left\langle R \gg\right.$ through $\langle K\rangle=\left\langle\left\langle R \gg / \varepsilon_{\mathrm{s}} q\right.\right.$ where $\varepsilon_{\mathrm{s}}$ is the extrapolated LS detection efficiency for ${ }^{222} \mathrm{Rn}$ in "unquenched" samples (given previously) and $q$ is a combined corrcction for the effects of radon losses (composition dependent) and chemical quenching in the cocktails. The product quantities $\varepsilon_{s} q$ are the experimentally-determined efficiencies $\varepsilon$ given, for example, in Fig. 1. From Eq. (1), the emanation fraction $f$ may then be obtained directly from determinations of $\langle K\rangle$ along with the decay-corrected ${ }^{226} \mathrm{Ra}$ source strength $\left[A_{0} \quad \exp \left(-\lambda_{\mathrm{Ra}} t_{\mathrm{d}}\right)\right], \quad{ }^{222} \mathrm{Rn}$ accumulation factor $\left[1-\exp \left(-\lambda_{\mathrm{R} n} t_{\mathrm{a}}\right)\right]$, and dilution mass $M$. The variability in replicate determinations of $f$ from evaluation run to run would then reflect the uncertainties in determining all of the above component quantities. 
The individual components of variance may be evaluated as follows. The overall measurement variability, given by $v_{\mathrm{C}}$ for $n_{\mathrm{m}}$ measurements on a single cocktail, may be considered to be related to the Poisson "counting error" $v_{P}$ through $v_{\mathrm{v}}^{2}=v_{\mathrm{P}}^{2}+n_{\mathrm{m}} v_{\mathrm{LS}}^{2}$ where $v_{\mathrm{LS}}$ is the relative standard deviation for an additional component of variance for the measurement process. Similarly, the overall variability between samples, given by $v_{R}$, can be considered to be comprised of the $v_{\mathrm{C}}$ component and an additional component of variance, given by a relative standard deviation $v_{\mathrm{S}}$, for the differences between samples: $v_{R}^{2}=v_{\mathrm{C}}^{2}+n_{\mathrm{s}} v_{\mathrm{S}}^{2}$. The variability in the mean $\langle K\rangle$ for a given generator run, given by a relative standard uncertainty $v_{K}$, is largely reflected in $v_{R}$ plus random variations in $\varepsilon_{\mathrm{s}}$ and in $q .{ }^{10}$ It follows that the observed variability in $n_{f}$ replicate determinations of $f$, given by a relative standard deviation $v_{f}$, can be related to $v_{K}$ (or $v_{R}$ ) plus an additional component for the variability among generator runs: $v_{f}^{2}=v_{K}^{2}+n_{f} v_{\mathrm{G}}^{2}$.

Hence, the following uncertainty components (all expressed as relative standard deviations) are derivable from the experimental evaluation measurements:

- $v_{P}$, the overall Poisson "counting error" for $n_{\mathrm{m}}$ multiple measurements of a single sample, obtained directly from the LS counting data for the samples and blanks;

- $v_{\mathrm{C}}$, the overall replication precision for $n_{\mathrm{m}}$ multiple measurements of $R$ for a single sample. computed from determinations of $R_{j, i}$ for a given sample $\mathrm{j}$ and having $\left(n_{\mathrm{m}}-1\right)$ degrees of freedom;

- $v_{\mathrm{LS}}$, the LS measurement precision for a single sample which arises from temporal instrument and cocktail instabilities, and from random variations in the ${ }^{222} \mathrm{Rn}$ decay corrections and livetime determinations, obtained from evaluations of $v_{P}$ and $v_{\mathrm{C}}$ and having $\left(n_{\mathrm{m}}-1\right)$ degrees of freedom;

- $v_{R}$, or $v_{K}$, the overall replication precision in determining $R$ for $n_{\mathrm{s}}$ multiple samples, computed from the mean $\left\langle R_{j}\right\rangle$ values and having $\left(n_{s}-1\right)$ degrees of freedom;

- $v_{\mathrm{S}}$, the sampling precision which arises from solution inhomogeneity (or sample-to-sample variability) and random variabilities in measuring the sample masses $m_{\mathrm{s}}$, obtained from evaluations of $v_{\mathrm{C}}$ and $v_{\mathrm{R}}$ and having $\left(n_{\mathrm{s}}-1\right)$ degrces of frccdom;

$-v_{f}$, the overall replication precision in determining $f$, computed from $n_{f}$ multiple determinations of $f$ having $\left(n_{f}-1\right)$ degrees of freedom; and

- $v_{G}$, the generator precision, which arises from random variabilities in determining the total dilution mass $M$ (primarily in turn arising from determining

\footnotetext{
${ }^{10}$ In actuality, $v_{K}$ is equivalent to the variability in $\left\langle R_{j} / m_{j}\right\rangle$ for constant $\varepsilon_{s} q$.
}

the "dead volume" $m_{\mathrm{d}}$ ) and in the decay-corrected

${ }^{226} \mathrm{Ra}$ source strength $A_{0}$ and the ${ }^{222} \mathrm{Rn}$ accumulation factor, obtained from evaluations of $v_{K}$ and $v_{f}$ and having $\left(n_{f}-1\right)$ degrees of freedom.

These uncertainty components evidently consist of a nested array. The components $v_{P}, v_{C}, v_{R}$ (or $v_{K}$ ) and $v_{f}$ are directly obtained from measurement data of replications; whereas $v_{\mathrm{LS}}$ is derived from $v_{P}$ and $v_{\mathrm{C}}, v_{\mathrm{S}}$ is derived from $v_{\mathrm{C}}$ and $v_{R}$, and $v_{\mathrm{G}}$ is derived from $v_{R}$ and $v_{f}$.

\section{Results of the performance evaluations}

The left-hand side of Fig. 2 gives the first four experimental determinations (labelled $\alpha 1, \alpha 2, \alpha 3$ and $\alpha 4$ ) of the emanation fraction $f$ when the generator was employed by following the original operating protocol [2]. In these cases, the total dilution was obtained by volumetric estimations and the "dead volume" was approximately $0.53 \mathrm{ml}$. All four determinations used comparable accumulation times $t_{\mathrm{a}}$ of 1 to 2 days, and were made over a relatively short two-week interval. The determinations were also based on roughly the same total dilution volume of $V=51.5-52.0 \mathrm{ml}$, the same number of samples $n_{\mathrm{s}}=7$ to $n_{\mathrm{s}}=10$ using sizes of $1.1 \mathrm{~g} \leq m_{j} \leq 1.5 \mathrm{~g}$ in $15 \mathrm{ml}$ of the same PCS scintillant, and each sample was counted an equivalent $n_{\mathrm{m}}=10$ number of times which resulted in very comparable Poisson "counting errors" $v_{p}$. The uncertainty intervals shown on the individual datum points in Fig. 2 reflect only the standard deviations $v_{R}$ in determining the $\langle R \gg$ values (defined above), and do not include any uncertainties in the total solution volume $V$ or mass $M$. The dispersion in the four determinations of $f$ (given by the standard deviation $v_{f}$ ) in comparison to the precision estimators $v_{R}$ clearly indicates that there is an additional and large component of uncertainty whose magnitude can not be attributed to uncertainties in either the decay-corrected ${ }^{226} \mathrm{Ra}$ content of the source or the ${ }^{222} \mathrm{Rn}$ accumulation factor [see Eq. (1) and previous discussion]. This additional uncertainty component can only be due to the inherent variability in the volumetric estimation of $V$ (or $M$ ).

This is evident on examination of the right-hand side of Fig. 2 which illustrates the next four experimental determinations (labelled $\omega 1, \omega 2, \omega 3$ and $\omega 4$ ) of $f$ that were obtained after the operating protocol was revised to more accurately reproduce and quantify the total dilution mass $M$ (as described previously). In these latter four cases (in which $M=m_{\mathrm{D}}+m_{\mathrm{d}} \simeq 57.57,56.08,57.58$ and $55.12 \mathrm{~g}$, respectively) the total mass of blank water $m_{\mathrm{D}}$ used to perform the dilution was gravimetrically determined (as obtained by summation of all masses $m_{j}$ dispensed in returning the dispensing syringe to its starting position) with an estimated relative standard uncertainty of approximately $0.15 \%$ and the reproducibility in $M$ was estimated to be better than $0.2 \%$. This 


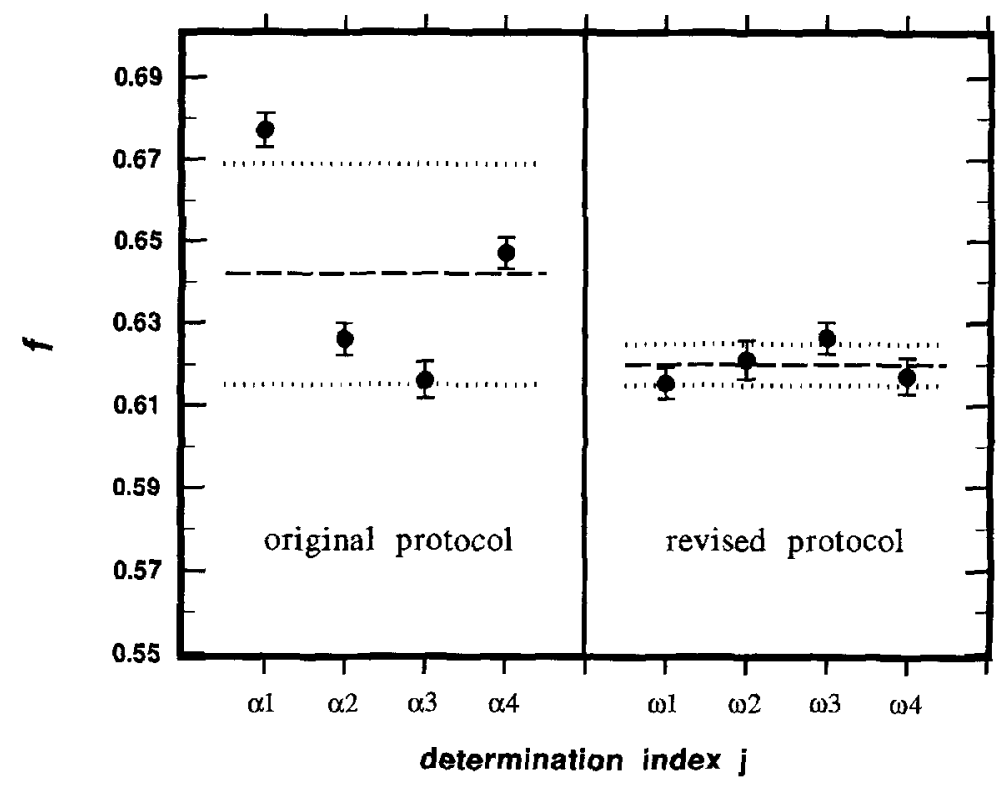

Fig. 2. Replicate determinations of the ${ }^{22} \mathrm{Rn}$ emanation fraction $f$ using the original volumetric-based operating protocol and a revised gravimetrically based protocol. The uncertainty interval bars on the individual datum values correspond to the standard deviations $f v_{k}$. The horizontal dashed and dotted lines, respectively, correspond to the two calculated mean $f$ values and their standard deviations $f^{\prime} f$.

may be contrasted with the relative uncertainty in determining $V$ (or $M$ ) using the original protocol which is now estimated to be greater than $\pm 1 \%{ }^{11}$ All other experimental conditions for determinations $o 1$ through $(\omega) 4$ were comparable and similar to those for $\alpha 1$ through $\alpha 4$ : $t_{\mathrm{a}}=1-3 \mathrm{~d} ; 8 \leq n_{\mathrm{s}} \leq 12$ cocktails per determination; $1.1 \mathrm{~g}$ $\leq m_{j} \leq 1.4 \mathrm{~g}$ in $15 \mathrm{ml}$ of PCS; and $n_{\mathrm{m}}=10-12$ measurements per cocktails. The relative uncertainties $v_{R}$ for each determination (given by the illustrated uncertainty interval bars in Fig. 2) were comparable for both the original and revised protocols. The relative standard deviation $v_{f}$, however, for the revised protocol is roughly 5 times smaller than that for the original protocol $v_{5}=0.8 \%$ compared to $v_{f}=4.2 \%$ ). All subsequent cvaluation data (and results) given here are based on use of this revised protocol.

Before addressing sample homogeneity (i.e., variation in ${ }^{222} \mathrm{Rn}$ concentration from sample to sample), it is useful to first consider the uncertainties associated with the LS measurements on any one sample. Fig. 3 provides estimates of the measurement precision (repeatability) $v_{\mathrm{C}}$ (defined previously) with $3 \leq n_{\mathrm{m}} \leq 20$ on each determination (as obtained from independent evaluations with 183 different cocktails from a variety of generator runs). These $v_{\mathrm{C}}$ values vary somewhat widely, from about $0.2 \%$

\footnotetext{
11 It is now believed that the originally reported relative uncertainty in determining $V$. given as $0.3 \%$ [2], was underestimated.
}

to well over $1 \%$, and with a mean of $0.75 \%$ and median of $0.73 \%$. The wide dispersion is just indicative of sampling $v_{\mathrm{C}}$ from a relatively broad $\chi^{2}$ distribution of $v_{\mathrm{C}}^{2}$. From evaluations of the total "counting error" $v_{P}$ on each determination, one can in turn estimate the additional uncertainty component $v_{\mathrm{LS}}$ (see above uncertainty model discussion). The determinations were chosen to cover a range $0.05 \% \leq v_{P} \leq 1.5 \%$. These 183 estimates of $v_{\mathrm{LS}}$ are also illustrated in Fig. 3, and have a mean of $0.24 \%$ and median of $0.23 \%$. It is apparent from the findings: that there is an additional component of variability $v_{\mathrm{LS}}$ in the LS measurement process (beyond that due to the "counting error" $v_{p}$ ); that the magnitude of this component is gencrally $v_{L S}=0.3 \%$; and that the overall measurement variability $v_{\mathrm{C}}$ for $n_{\mathrm{m}}$ measurements on a single cocktail can be approximated by $v_{C} \simeq\left(v_{\vec{P}}^{2}\right.$ $\left.+n_{\mathrm{m}} v_{\mathrm{LS}}^{2}\right)^{1 / 2}$, which has a corresponding standard deviation of the mean $v_{\mathrm{C}} / \sqrt{ } n_{\mathrm{m}}$ which is generally in the range $0.3-0.4 \%$ for most determinations with $v_{P} \leq 0.1 \%$. The estimates of $v_{\mathrm{LS}}$ were invariant of the magnitude of $v_{P}$, i.e., the $v_{P}$ and $v_{\mathrm{LS}}$ components of $v_{\mathrm{C}}$ were uncorrelated. The magnitude of these $v_{C}$ and $v_{L S}$ uncertainty components comport well with those found in other measurements with our LS systems [18-22].

Homogeneity (or between-sample variability) was originally estimated to be the largest contributing source of uncertainty in the ${ }^{222} \mathrm{Rn}$ concentration in a given dispensed aliquant. This uncertainty component was presently evaluated in two ways: first, by consideration of the variation in measured $R_{j} / m_{j}$ values for a set of samples 


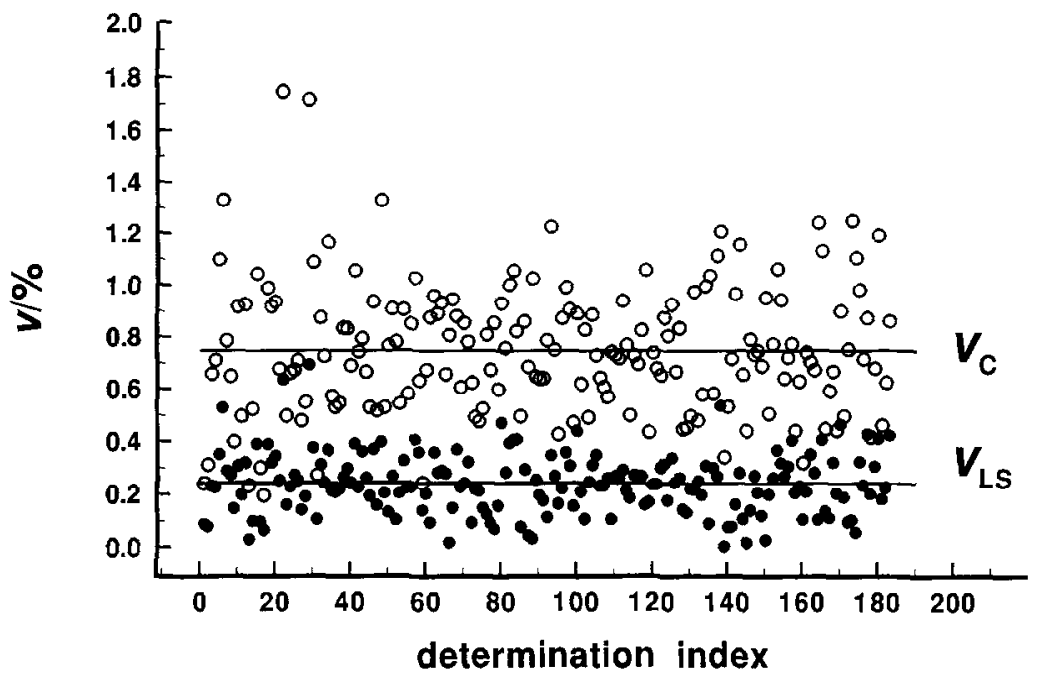

Fig. 3. Evaluations of the relative uncertainty components (in $\%$ ) for the overall replication precision $v_{\mathrm{C}}$ for $3 \leq n_{\mathrm{m}} \leq 12$ multiple measurements of a single cocktail and the LS measurement precision $v_{\mathrm{LS}}$ (as derived from 183 independent estimates of $v_{\mathrm{C}}$ and the total "counting error" ${ }^{\prime} p$ ) . Refer to text. The relative standard deviation of the mean $v_{\mathrm{C}} / \sqrt{ } n_{\mathrm{m}}$ for any given cocktail is typically $0.3-0.4 \%$.

obtained from any given generator run; and second, by consideration of $R_{j} / m_{j}$ when the size of the aliquant $m_{j}$ varied over a fairly large range. Additionally, it is evident that these variations would, of course, depend upon the adequacy of the mixing procedure in the operating protocol.

Now, in the first case, one can estimate the homogeneity from an evaluation of $v_{\mathrm{S}}$ as obtained from determinations of $v_{K}$ and $v_{C}$ (using an analysis similar to that given above for obtaining $v_{\mathrm{LS}}$ from $v_{p}$ and $v_{\mathrm{C}}$. Table 3 gives the results for $v_{\mathrm{s}}$ as obtained from 11 generator runs (each with cocktails having $f_{\mathrm{w}} \simeq 0.1$ and $m_{\mathrm{c}} \simeq 20 \mathrm{~g}$ ). Fach run had a computed $v_{K}$ (or $v_{R}$ ) for $n_{\mathrm{s}}$ dispensed samples (as tabulated). From the mean $v_{\mathrm{C}}$ obtained by averaging $v_{\mathrm{C}(j)}$ across all samples within a given run (with each individual $v_{\mathrm{C}, j}$ computed from the results of $n_{\mathrm{m}}$ measurements on each sample), one can estimate the $i_{\mathrm{s}}$ for that run. As expected, $v_{K}$ and $v_{\mathrm{S}}$ vary widely (again, considering the statistical sampling from broad $\chi^{2}$ distributions of $v_{\mathrm{K}}^{2}$ and $v_{\mathrm{S}}^{2}$ ). For illustrative purposes, Fig. 4 gives relevant data for the first two runs. In Fig. 4(a), the computed $v_{K}$ for the 9 dispensed samples was $v_{K} \simeq 1.2 \%$. The mean $v_{\mathrm{C}}$ for the 9 samples was $v_{\mathrm{C}} \simeq 0.67 \%$ (with median $v_{\mathrm{C}} \simeq 0.69 \%$ ) which yields an estimate of $v_{\mathrm{s}} \simeq 0.33 \%$. In Fig. $4(\mathrm{~b})$, the computed estimates of $v_{K}$ and $v_{C}$ are virtually equal, yielding an estimate of $v_{\mathrm{S}} \simeq 0 .{ }^{12}$

In the second case, Fig. 5 illustrates typical results for the relative invariance in the derived massic activity

\footnotetext{
${ }^{12}$ In fact, $v_{\mathrm{S}}$, in this case, is indeterminate with a magnitude of imaginary number (square root of a negative number) since the estimates of $v_{K}$ and $v_{C}$ had $v_{C}>v_{K}$.
}

Table 3

Evaluation of the relative standard deviation $v_{\mathrm{S}}($ in $\%$ ) for the sampling precision (homogeneity) as obtained from estimates of the relative standard deviations $v_{K}$ and $v_{C}$ in 11 generator runs

\begin{tabular}{lllrl}
\hline & $v_{K}(\%)$ & Mean $v_{C}(\%)$ & $n_{\mathrm{s}}$ & $v_{\mathrm{S}}(\%)$ \\
\hline & 1.2 & 0.73 & 9 & 0.33 \\
0.74 & 0.75 & 7 & - \\
& 1.5 & 0.20 & 7 & 0.56 \\
& 1.0 & 0.23 & 7 & 0.38 \\
& 2.2 & 0.32 & 10 & 0.69 \\
& 0.93 & 0.65 & 10 & 0.21 \\
& 1.8 & 0.72 & 9 & 0.54 \\
& 1.4 & 0.85 & 7 & 0.40 \\
& 2.5 & 0.65 & 9 & 0.80 \\
Median & 1.3 & 0.44 & 9 & 0.41 \\
Mean & 2.3 & 0.53 & 10 & 0.71 \\
& 1.4 & 0.65 & 9 & 0.47 \\
\hline
\end{tabular}

$K$ (proportional to $R_{j} / m_{j}$ ) obtained with cocktails having a large range of $m_{j}$. Each cocktail for this sample series was prepared with near constant $f_{\mathrm{w}} \simeq 0.34$ (by the addition of a variable quantity of blank $\mathrm{H}_{2} \mathrm{O}$ plus $m_{j}$ to $\simeq 13 \mathrm{~g} \mathrm{PCS}$ ) and $m_{\mathrm{c}} \simeq 20 \mathrm{~g}$. The $m_{j}$-varying aliquants were dispensed in order of increasing $m_{j}$. As indicated there are no substantial differences in $K$ between cocktails prepared initially with small aliquant sizes $\left(m_{j}<1 \mathrm{~g}\right)$ and with later ones having large multiple-gram aliquants. For comparative purposes with Table $3, v_{K}$, in this case, was $v_{K} \simeq 0.95 \%$ and the mean $v_{\mathrm{C}}$ (across all 25 cocktails) was $v_{\mathrm{C}} \simeq 0.47 \%$ (with median $v_{\mathrm{C}} \simeq 0.45 \%$ ), which yields an estimate of $v_{\mathrm{S}} \simeq 0.2 \%$. 

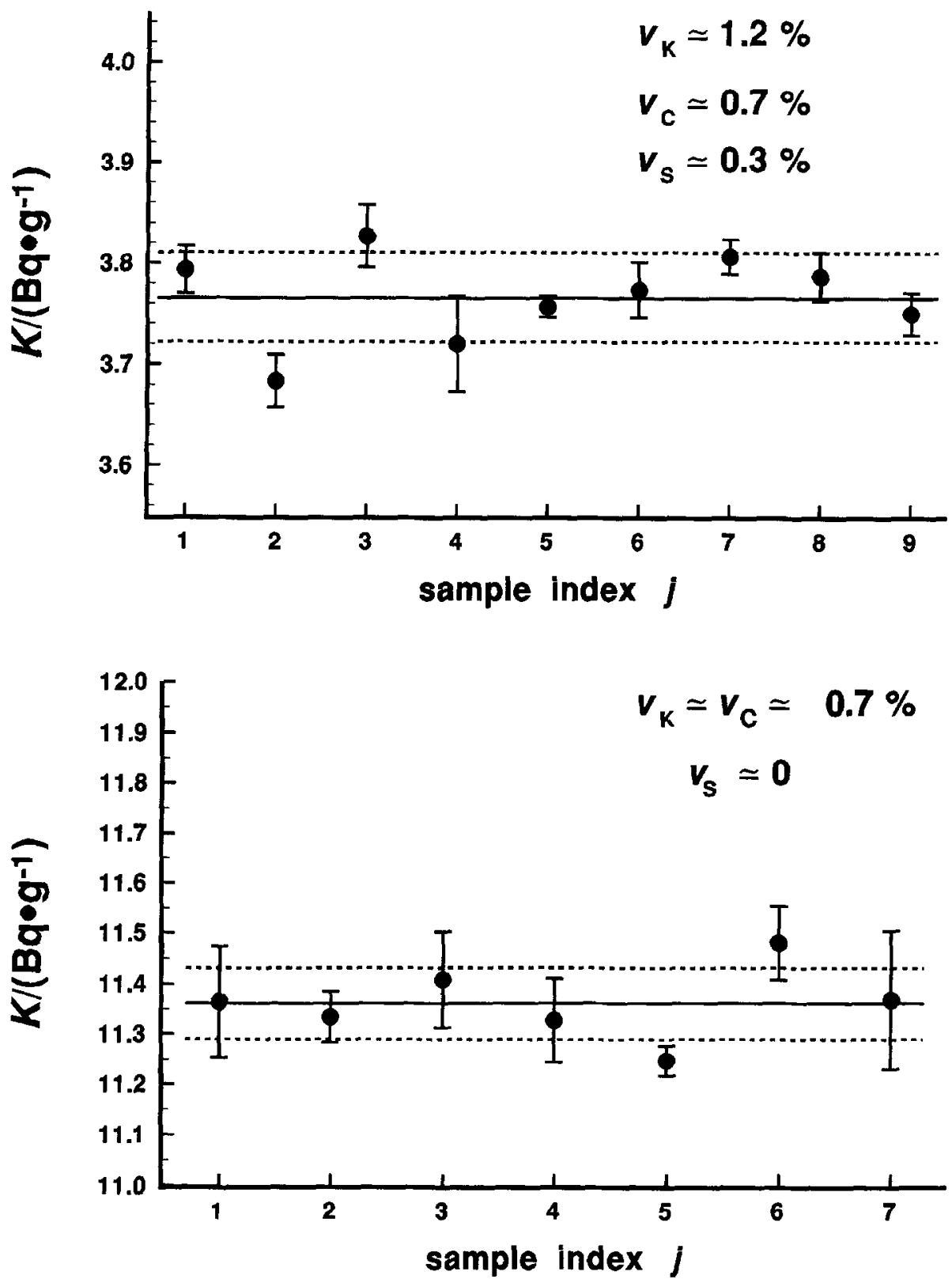

Fig. 4. Representative data for the LS-assayed ${ }^{222} \mathrm{Rn}$ activity concentration $K$ (in units of $\mathrm{Bq} \mathrm{g}^{-1}$ ) in a series of dispensed aliquants with near constant $m_{j}$ as obtained in two different generator runs (with $n_{\mathrm{s}}=9$ (upper) and $n_{\mathrm{s}}=7$ (lower) aliquants, respectively). The uncertainty interval on each datum corresponds to the standard deviation $K v_{\mathrm{c}}$ for $n_{\mathrm{m}}$ replicate LS measurements on the given aliquant. The solid and dashed horizontal lines, respectively, correspond to the mean $K$ obtained for the run and its standard deviation $K v_{K}$ interval for the $n_{\mathrm{s}}$ samples. The "sampling precision" $v_{\mathrm{S}}$ is derived from estimates of $v_{C}$ and $v_{K}$ (refer to text).

This same sample series could also be used to evaluate, very thoroughly, the conversion factor $k_{\mathrm{x}}$ for the dispensed mass of an aliquant per turn of the dispensingsyringe motordrive. Each aliquant was dispensed with a given number (or fraction) of turns and had a gravimetrically determined $m_{j}$ for that aliquant. Hence, the conversion factor $k_{\mathrm{x}}$ could be evaluated for each dispensing
(Fig. 6). The mean $k_{\mathrm{x}}$ and standard deviation from this generator run (obtained with widely varying $m_{j}$, i.e., with $\frac{1}{4}$ to 7 turns) were $k_{\mathrm{x}}=0.7840 \pm 0.0012 \mathrm{~g}$ (per turn). This precision is not typical, however, since extraordinary care was taken during this run to very precisely control the number of motordrive turns (particularly for the fractional numbers). Other evaluations of $k_{\mathrm{x}}$ from additional, 


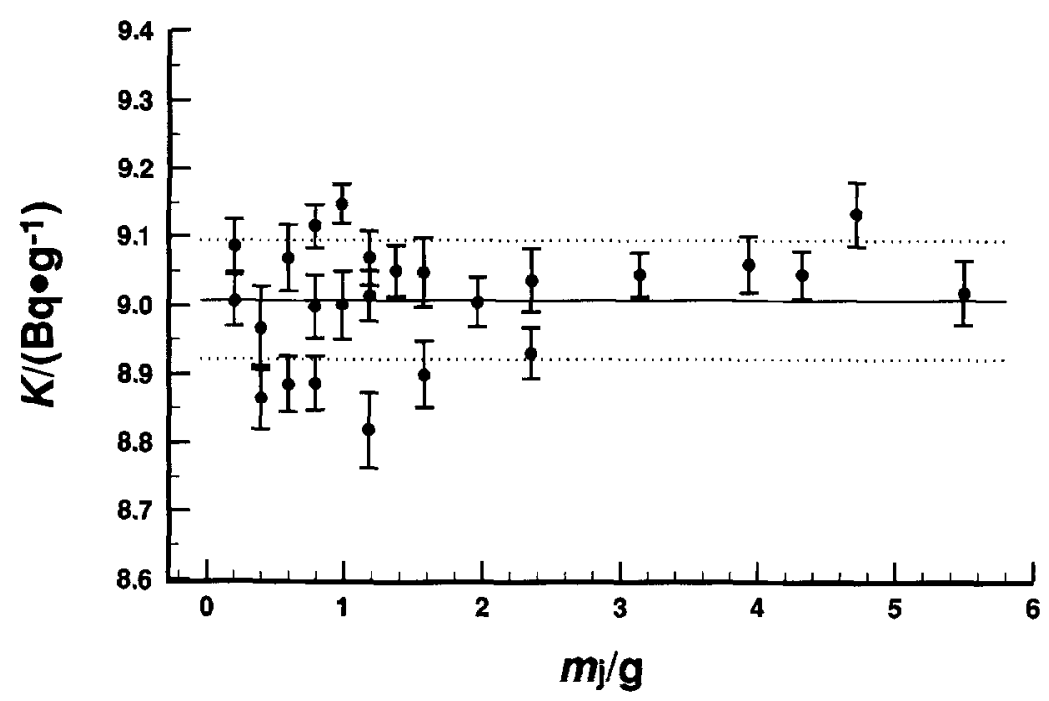

Fig. $5 .{ }^{222} \mathrm{Rn}$ activity concentration $K$ (in units of $\mathrm{Bqg}^{-1}$ ) as a function of dispensed aliquant mass $m_{j}$ (in $\mathrm{g}$ ) over a large $m_{j}$ range. The uncertainty interval on each datum corresponds to the standard deviation $K v_{\mathrm{C}}$ for $n_{\mathrm{m}}$ replicate LS measurements on the given aliquant. The solid and broken horizontal lines, respectively, correspond to the mean $K$ obtained for the run and its standard deviation $K v_{K}$ interval for the $n_{\mathrm{s}}$ samples.

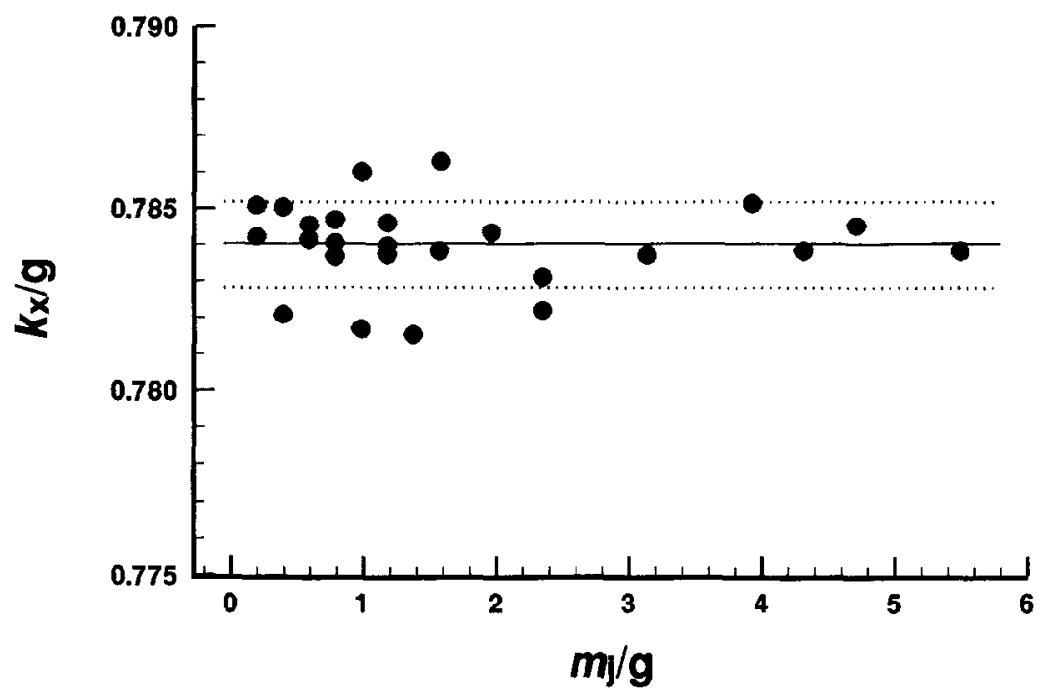

Fig. 6. Evaluation of the conversion factor $k_{\mathrm{x}}$ for the mass $m_{j}$ (in units of g) of a dispensed aliquant per number of turns on the dispensing-syringe motordrive. The horizontal solid and dashed lines correspond, respectively, to the mean $k_{\mathrm{x}}$ and its standard deviation interval $k_{\mathbf{x}} v_{\mathbf{k x}}$.

more typical, generator runs are summarized in Table 4. The relative standard deviation $v_{k \mathrm{x}}$ computed on the grand mean $k_{\mathrm{x}}=0.7810 \mathrm{~g}$ from the 10 generator runs was $v_{\mathrm{kx}} \simeq 0.92 \%$. Invariably, the uncertainty in $k_{\mathrm{x}}$ (from a particular generator run) is decidedly smaller when the aliquant dispensings have comparable $m_{j}$ (i.e., are dispensed with equal numbers of turns), have larger $m_{j}$, or have an integral (non-fractional) number of turns. The uncertainty in $k_{\mathbf{x}}$ for a typical, routine operation (across all $m_{j}$ sizes) can be characterized in terms of a relative standard deviation of the mean $v_{\mathrm{m}}\left(k_{\mathrm{x}}\right)$ obtained from the quadratic combination of $v_{k x}$ and a typical relative standard deviation of the mean $v_{\mathrm{kx}(i)} / \sqrt{n_{\mathrm{s}(i)}}$ for any given generator operation; i.e., $v_{\mathrm{m}}\left(k_{\mathrm{x}}\right)=\left[v_{\mathrm{kx}}^{2}+\right.$ $\left.\left(v_{k \mathrm{x}(i)} / \sqrt{n_{\mathrm{s}(i)}}\right)^{2}\right]^{1 / 2}$ since the between-run variability $v_{k \mathrm{x}}$ is not homogeneous with the within-run variability $v_{k x(i)}$. The typical $v_{k x(i)}$ may be taken to be the mean (or median) obtained from averaging across all 10 runs, which is 
Table 4

Evaluation of the conversion factor $k_{\mathrm{x}}$ for the mass (in units of g) of dispensed solution per turn of the syringe motordrive as obtained in 10 generator runs. Each tabular $k_{x}$ is that obtained from a given run with $n_{\mathrm{s}}$ dispensed aliquants having a range $n_{\mathrm{turn}}$. The uncertainty estimator $v_{k x i} / \sqrt{n_{s}(i)}$ is the relative standard deviation of the mean (in \%) for the given generator run

\begin{tabular}{lrll}
\hline$n_{\text {turn }}$ range & $n_{\mathrm{s}}$ & $k_{\mathrm{x}}(\mathrm{g})$ & $\left(c_{\left.\mathrm{hx}(i) / \sqrt{ } / n_{\mathrm{s}(i)}\right)(\%)}\right.$ \\
\hline $0.25-7$ & 25 & $0.7840 \pm 0.0012$ & 0.031 \\
$0.25-7$ & 8 & $0.7701 \pm 0.0159$ & 0.73 \\
$0.25-2.25$ & 10 & $0.7752 \pm 0.0231$ & 0.94 \\
$0.25-1.5$ & 7 & $0.7758+0.0477$ & 2.3 \\
$0.5-2$ & 7 & $0.7725 \pm 0.0208$ & 1.0 \\
$2-8$ & 7 & $0.7885 \pm 0.0041$ & 0.20 \\
$1-5$ & 9 & $0.7900 \pm 0.0081$ & 0.34 \\
$1-6$ & 15 & $0.7800 \pm 0.0140$ & 0.46 \\
$1-4$ & 12 & $0.7875 \pm 0.0063$ & 0.23 \\
$1-5$ & 9 & $0.7860 \pm 0.0067$ & 0.28 \\
Grand mean & & $0.7810 \pm 0.0072$ & 0.65 \\
\hline
\end{tabular}

$0.65 \%$. The propagation of $v_{k x} \simeq 0.95 \%$ and typical $v_{k x(i)} \simeq 0.65 \%$ results in an estimate $v_{\mathrm{m}}\left(k_{\mathrm{x}}\right) \simeq 1.1 \%$.

To homogenize the ${ }^{222} \mathrm{Rn}$ solution prior to dispensing, the original operating protocol [2] utilized (and was found to require) two complete transfers of the solution between the two syringes. The transfers may be more clearly understood by reference to the schematic layout of the generator as given by Hutchinson, et al. [2]. The diluent blank $\mathrm{H}_{2} \mathrm{O}$ (after passing through the accumulation chamber) first filled the left-hand side syringe (L). The ${ }^{22} \mathrm{Rn}$-laden solution in $\mathrm{L}$ was then transferred to the right-hand side syringe ( $R$ ), then back to $L$, back to $R$, and finally back to $L$ for initiation of any dispensing. This invoked operation constituted (by definition) two complete mixing transfers, and was believed to be sufficiently adequate for obtaining a desired degree of solution homogeneity $(<1 \%$ differences amongst aliquants). The adequacy of this mixing procedure was re-examined in the present evaluations. Fig. 7 shows the variation in $v_{K}$ and $v_{s}$ as a function of the number $n_{x}$ of mixing transfers. The conditions for each generator run were comparable with $7 \leq n_{s} \leq 9$ cocktails each containing approximately $m_{j} \simeq 1.5 \mathrm{~g}$ aliquants in $18.5 \mathrm{~g}$ of RS scintillant. As before, $v_{\mathrm{s}}$ was estimated from the computed $v_{K}$ for each generator run and the mean $v_{C}$ obtained by averaging over all cocktails for the run. The results for $v_{\mathrm{s}}$ [Fig. 7(b)] suggest that there is a slight improvement in solution homogeneity with $n_{\mathrm{x}}=3$ compared to that obtained with $n_{\mathrm{x}}-2$. The improvement however is very slight. Nevertheless, the operating protocol was revised in late 1992 to routinely employ $n_{\mathrm{x}}=3$ (rather than $n_{\mathrm{x}}=2$ ) mixing transfers.

The most critical parameter for the generator, the ${ }^{222} \mathrm{Rn}$ emanation fraction $f$, was independently evaluated many times over the period from February 1989 to
March 1995. The results for $K$ (for various generator runs) previously presented here constituted some of these $f$ evaluations. The 26 evaluations of $f$ considered here were obtained under a broad array of variable conditions. These include: (1) a ${ }^{226} \mathrm{Ra}$ source with varying "age" (i.e., the time interval between its initial preparation and the start of the accumulation for a given run) ranging from 0.3 a to $6.3 \mathrm{a}$; (2) variable accumulation times $0.9 \mathrm{~d} \leq t_{\mathrm{a}}>30 \mathrm{~d}$; (3) somewhat constant, but nevertheless variable total dilution mass $53 \mathrm{~g} \leq M \leq 58 \mathrm{~g}$ (gravimetrically determined for each run): (4) variable numbers of mixing transfers $2 \leq n_{x} \leq 5$, although the first 19 of the 26 determinations were obtained with $n_{\mathrm{x}}=2$; (5) variable numbers of dispensed aliquants for a given generator run ranging from as few as $n_{\mathrm{s}}=6$ to $n_{\mathrm{s}}>20$; (6) prepared cocktails containing near constant $m_{j}$ aliquants for a given run, as well as with variable $0.2 \mathrm{~g}$ $\leq m_{j} \leq 5 \mathrm{~g}$ within a run; (7) varying $f_{\mathrm{w}}$ and $m_{\mathrm{c}}$ cocktail compositions, although most of these $f$ determinations were obtained with $f_{\mathrm{w}}>0.07$ and $m_{\mathrm{c}} \simeq 20 \mathrm{~g}$ : (8) cocktails prepared with three different scintillants (Table 2); (9) variable numbers of LS measurements performed on each cocktail tanging $5 \leq n_{\mathrm{m}} \leq 120$, but generally with $v_{p}<0.1 \%$; (10) LS measurements performed over time intervals of $2 \mathrm{~d}>t_{i}>12 \mathrm{~d}$ [see Eq. (2)]; and (11) use of two different spectrometers for the measurements (Table 1). All of the $f$ evaluations considered here utilized the "flushing" procedure given in the initial protocol [2] and the revised procedure for determining $M$ by increasing the "dead volume" $m_{\mathrm{d}}$ and gravimetrically determining $M_{D}$ (as described above). A majority of these $f$ determinations were obtained from runs performed in the first 2 years of operation (i.e., up to 1991).

Fig. 8 gives the results of these $26 \mathrm{f}$ evaluations which have a mean value of $f=0.6255$. The uncertainty bars on each datum correspond to the computed $v_{K}$ for that run. The mean $v_{K}$ across all 26 runs was $r_{K} \simeq 1.29 \%$ (with median $v_{k} \simeq 1.32 \%$ ). The overall reproducibility in $f$ (in terms of the relative standard deviation $v_{f}$ ) from the 26 evaluations was $v_{f} \simeq 1.55 \%$. With these estimates of $v_{K}$ and $v_{j}$, one can approximate the previously defined "generator precision" $v_{\mathrm{G}}$ to be $0.2 \%$. Re-examination of Fig. 2, alongside Fig. 8, clearly demonstrates the vastly improved precision obtained in going to the protocol revisions. These results also support the conclusion that the total dilution mass $M$ can be determined to a precision of a few tenths of a percent, but that the uncertainty in the volume-based dilutions (for the original protocol [2]) was seriously underestimated previously.

Scientific instinct dictates that two of the more likely variables that may affect $f$ are the "age" of the ${ }^{226} \mathrm{Ra}$ capsule $t_{\text {age }}$ and the accumulation time $t_{\mathrm{a}}$, Fig. 9 demonstrates the invariance in $f$ with both variables. Fig. $9(\mathrm{~b})$ includes some additional points with $t_{\mathrm{a}}<1 \mathrm{~d}$ not included in the data of Fig. 8 . For these comparisons, it must be emphasized that other variables were not 

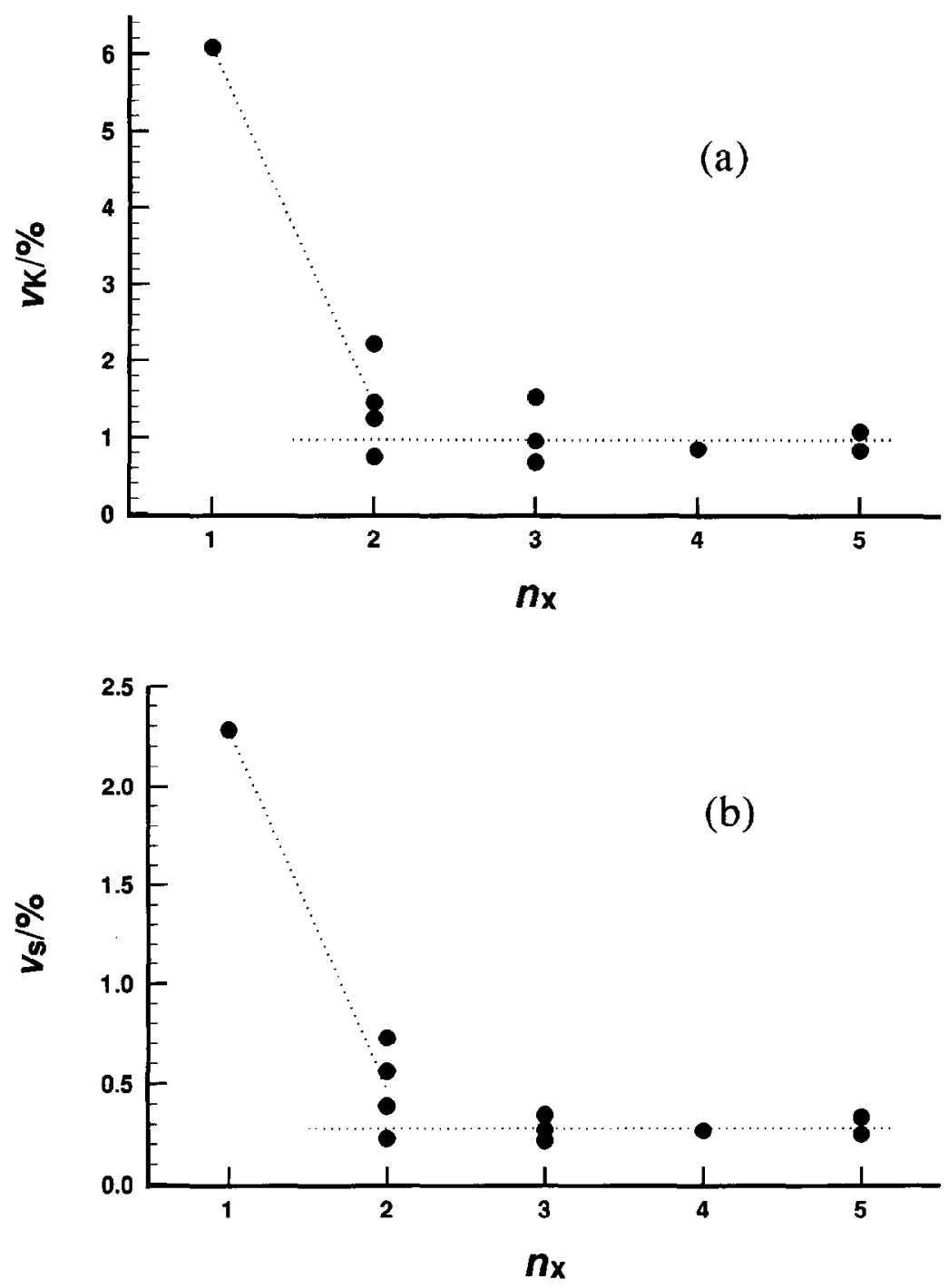

Fig. 7. Variation in the uncertainty estimators $v_{k}$ and $v_{\mathrm{S}}$ as a function of the number of syringe-transfer mixes $n_{x}$.

necessarily held constant (nor were they completely randomized). In particular, the invariance of $f$ with $t_{\text {age }}$ was obtained under systematic conditional changes. All initial $f$ determinations (at young $t_{\text {age }}$ ) were obtained by operating the generator with only $n_{\mathrm{x}}=2$ mixings. The dispensed aliquants from these initial runs formed aliquants with only the PCS scintillant (Table 2) that were measured only with the system B spectrometer (Table 1). In contradistinction, the more recent $f$ determinations were obtained with $n_{\mathrm{x}}>3$ mixings and utilized cocktails that were formed with IG and RS scintillants that were in turn measured with both the $\mathrm{B}$ and $\mathrm{P}$ spectrometers. In addition, the initial air void in the ${ }^{226} \mathrm{Ra}$ capsule (mentioned previously) also continuously diminished with time.

Equally, the temperature of the ${ }^{222} \mathrm{Rn}$ accumulations (as it may affect the solubility and diffusion of radon out of the polyethylene capsule and into the water of the accumulation chamber) was uncontrolled (and largely unmonitored). The $f$ evaluations were performed, however, during all seasons which have known ambient temperature variations ranging from about $17^{\circ} \mathrm{C}$ to $25^{\circ} \mathrm{C}$. The experimentally-determined (and empirically-defined) $f$ for the generator is thereby considered to be valid for a temperature range of $21 \pm 3^{\circ} \mathrm{C}$. Inasmuch as the encapsulated ${ }^{226} \mathrm{Ra}$ source is wholly immersed in the $\mathrm{H}_{2} \mathrm{O}$ of the accumulation chamber and that it appcars to act as a purely-diffusive device (with no permeation component) [2], effects due to ambient humidity or atmospheric pressure changes are believed to be non-existent or negligible.

Finally, three other evaluations were also performed. One was to test the adequacy of the "flushing" procedure 


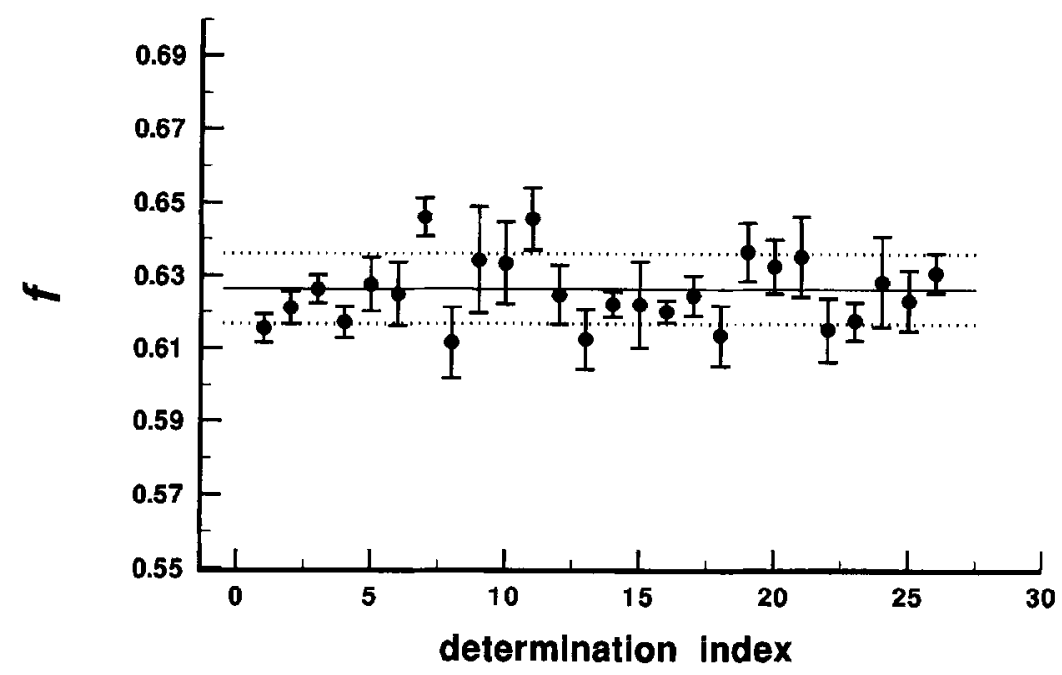

Fig. 8. Evaluation of the ${ }^{222} \mathrm{Rn}$ emanation fraction $f$ for the Radon-In-Water Standard Generator as obtained from 26 evaluations over a period of about 6 years under many variable conditions (see text). The horizontal solid and broken lines correspond, respectively, to the mean $f$ and its standard deviation interval $f v_{f}$

to ensure proper initialization conditions for an accumulation (i.e., that the steady-state boundary conditions for the diffusion of radon out of the capsule were reproducibly achieved). The flushing is performed to obtain, at the start of an accumulation $\left(t_{\mathrm{a}}=0\right)$, a virtual zero ${ }^{222} \mathrm{Rn}$ concentration in the watcr contained in the accumulation chamber, and a steady-state diffusive flux of ${ }^{222} \mathrm{Rn}$ out of the polyethylene. The tests were performed by varying both the flushing duration and volume flow rate of rinse $\mathrm{H}_{2} \mathrm{O}$ and examining the predicted accumulated ${ }^{222} \mathrm{Rn}$ activity for relatively shorl $\left(0.3 \mathrm{~d}<t_{\mathrm{a}}<1 \mathrm{~d}\right)$ accumulations. The extant protocol [2] (which consists of an initial rinse of about $50 \mathrm{ml}$ of "radon-free" $\mathrm{H}_{2} \mathrm{O}$, followed by continuous flushing at a flow rate of about $0.1 \mathrm{lh}^{-1}$ for a minimum of $6 \mathrm{~h}$ to remove any excess ${ }^{222} \mathrm{Rn}$ dissolved in the polyethylene, and finally by a $50 \mathrm{ml}$ rinse that is performed as rapidly as possible) was found to be wholly adequate. A second evaluation was to independently assess the reproducibility in determining the "dead volume" $m_{\mathrm{d}}$ (i.e., the reproducibility in returning the syringes to their starting positions) by replicate measurements of the dilution mass $m_{\mathbf{D}}$ under conditions of near constant $m_{\mathrm{d}} \simeq 1.55 \mathrm{~g}$ and $M=m_{\mathrm{d}}+m_{\mathrm{D}} \simeq 55 \mathrm{~g}$. This test was intended to independently verify the uncertainty estimator $v_{G}$ (which contains the uncertainty in determining $M$ ) and in estimating the uncertainty on $m_{\mathrm{d}}$. Gravimetric determinations of $m_{\mathbf{D}}$ from six closely-controlled trials resulted in a relative standard deviation of $v\left(m_{\mathrm{D}}\right) \sim$ $0.14 \%$. With $v_{\mathrm{M}}=\left[\left(m_{\mathrm{d}} / M\right)^{2} v\left(m_{\mathrm{d}}\right)^{2}+\left(m_{\mathrm{D}} / M\right)^{2} v\left(m_{\mathrm{D}}\right)^{2}\right]^{1 / 2}$ and assuming that $v_{\mathrm{G}} \simeq 0.2 \%$ is wholly due to the relative standard deviation $v_{\mathrm{M}}$, one obtains an estimate of $v\left(m_{\mathrm{d}}\right) \simeq 5.2 \%$ (which corresponds to an uncertainty in $m_{\mathrm{d}}$ of about $\pm 0.08 \mathrm{~g}$ ). This comports reasonably well with our geometrically-based estimate of $m_{\mathrm{d}} \simeq$
$1.55 \pm 0.15 \mathrm{~g}$. A third evaluation was to routinely test for possible ruptures or leakage losses of ${ }^{226} \mathrm{Ra}$ from the capsule. This was rather easily performed by LS measurements of cocktails (containing large-volume dispensed aliquants from the generator) after either long ${ }^{222} \mathrm{Rn}$ decay-time intervals ( $>40 \mathrm{~d}$ ) or after purging the ${ }^{222} \mathrm{Rn}$ from the cocktails and merely waiting for the short-lived ${ }^{214} \mathrm{~Pb}-{ }^{214} \mathrm{Bi}-{ }^{214}$ Po subseries to decay ( $>6 \mathrm{~h}$ ). No losses of ${ }^{226} \mathrm{Ra}$ from the capsule were ever detected, thereby ensuring the integrity of the source and that the ${ }^{222} \mathrm{Rn}$ solutions obtained from the generator (and subsequent ${ }^{222} \mathrm{Rn}$ calibrations) are indeed "radium free".

A considerable number of additional statistical analyses beyond those reported here were performed on the vast data set obtained from these evaluations. They included: (1) sequential time-series analyses to determine if there were any time dependencies or correlations in the order of samples or order of measurements for the various evaluated parameters (e.g., $R_{j} / m_{j}$ for a given cocktail within a $n_{\mathrm{m}}$ measurement sequence) or in the various statistical estimators (e.g., $v_{C}$ or $v_{K}$ ); (2) regression analyses between various combinations of variables (e.g., $R_{j} / m_{j}$ versus $m_{j}$ for cocktails within a given run or for $f$ versus $M$ between runs); (3) divisions of the data (e.g., for quantities like $R_{j} / m_{j}, v_{K}$, or $f$ ) into classes (e.g., those obtained with one scintillant compared to those obtained with the other scintillants, or with one spectrometer versus the other) and testing the resulting subsets of data for differences in the various means using $t$-tests, and for the homogeneity in the various subset means and variances using $\chi^{2}$ - and $F$-tests; and (4) sequential two-variable analysis-of-variance (ANOVA) techniques for any differences in similarly constructed subset means and variances. None of the tests (excepting the $m_{j}$ and 

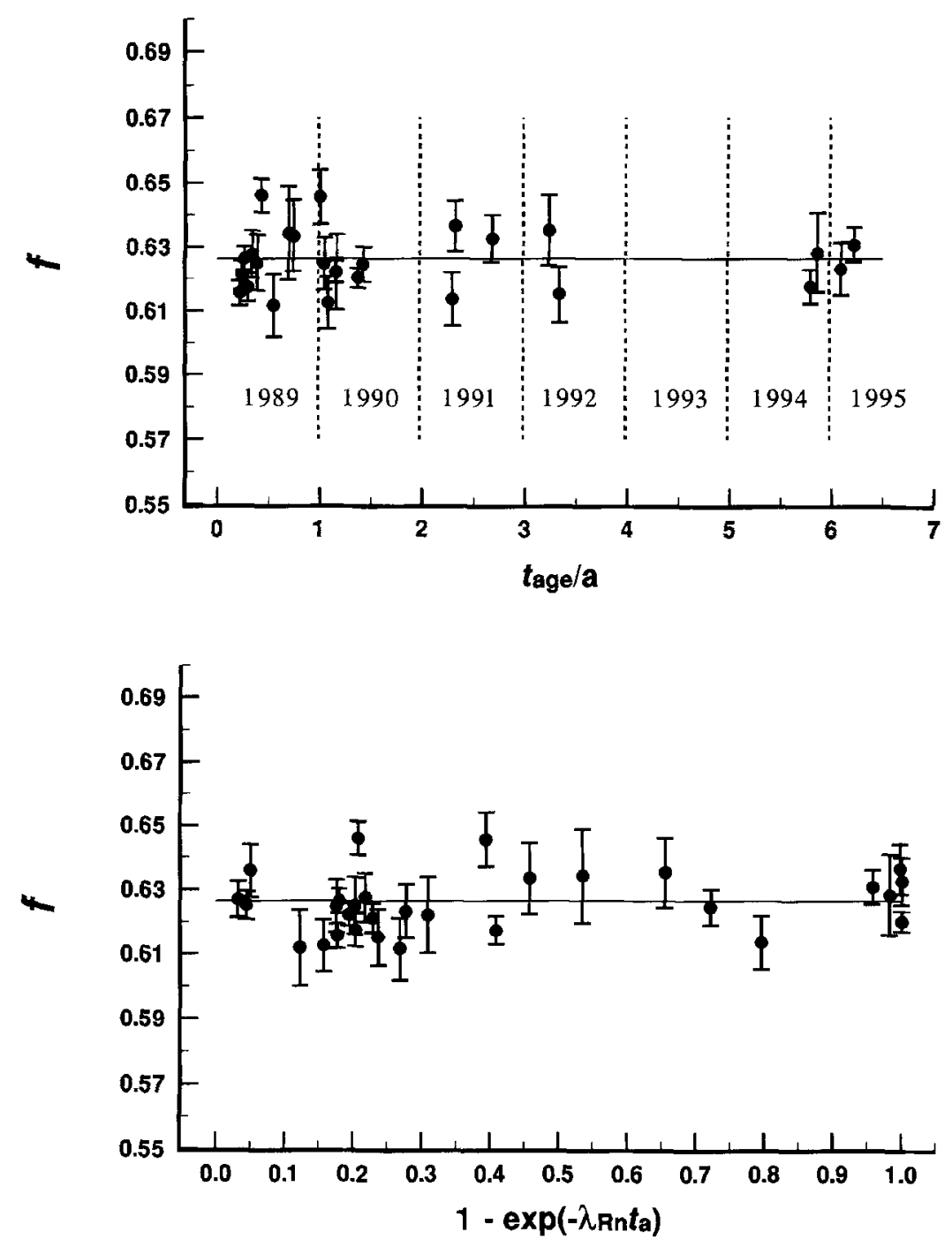

Fig. 9. ${ }^{222} \mathrm{Rn}$ emanation fraction $f$ as functions of the "age" $t_{\text {age }}$ (in units of a) of the ${ }^{226} \mathrm{Ra}$ encapsulated source (upper trace) and the accumulation factor $\left[1-\exp \left(-\lambda_{\mathrm{Rn}} t_{\mathrm{a}}\right)\right]$ for ${ }^{222} \mathrm{Rn}$ growth and accumulation (lower trace).

fractional-turn dependencies on $k_{\mathrm{x}}$ noted above) indicated that there were any statistically significant differences in any of the time dependencies. correlations, or differences in the tested subset sample means and variances.

\section{Generator parameters and uncertainty assessments}

Uncertainty cvaluations nced to consider two distinct assessments. Firstly, there is the assessment needed to evaluate the uncertainties associated with the parameters for the generator's operation. Secondly, and more importantly, is the assessment needed to assign a realistic uncertainty to the total ${ }^{222} \mathrm{Rn}$ activity content $A$ or activity concentration $K$ in an aliquant dispensed from the generator (when appropriately derived by rigorously invoking the operating protocol). In addition to the statistically evaluated uncertainties (given above), additional uncertaintics requiring evaluation are those associated with the activity of the ${ }^{226} \mathrm{Ra}$ source, with the mass determinations, and with the radioactive decay and accumulation $\left({ }^{222} \mathrm{Rn}\right.$ growth) factors.

The statistically-evaluated uncertainties $v_{P}, v_{\mathrm{C}}, v_{\mathrm{LS}}$, $v_{R}$ (or $\left.v_{\mathrm{K}}\right), v_{\mathrm{S}}, v_{f}, v_{\mathrm{G}}\left(\mathrm{or} v_{\mathrm{M}}\right)$, and $v_{\mathrm{m}}\left(k_{\mathrm{x}}\right)$ (as defined previously) can be summarized in a very general way as given in Table 5. The quality of these estimates can be characterized in terms of either the degrees of freedom associated with each (given previously) or the variability obtained from replicate evaluations (i.e., the "uncertainty in the uncertainty") of the same estimator (e.g., the 
Table 5

Summary of the statistically evaluated uncertainties obtained from the present evaluations of the Radon-In-Water Standard Generator

\begin{tabular}{ll}
\hline Estimator & Relative standard deviation \\
\hline$v_{P}$ & variable, but generally $v_{P}<0.2 \%$ \\
$v_{\mathrm{C}}$ & Largely depends on $n_{\mathrm{m}}\left(\right.$ and $\left.v_{P}\right)$, but generally \\
& $v_{\mathrm{C}} / \sqrt{n_{\mathrm{m}}} \simeq 0.4 \%$ \\
$v_{\mathrm{LS}}$ & $0.2 \%$ \\
$v_{R}\left(\right.$ or $\left.v_{K}\right)$ & Largely depends on $n_{\mathrm{s}}$ (and $v_{\mathrm{C}}$ ), but generally \\
& $v_{\mathrm{K}} / \sqrt{n_{\mathrm{s}}} \simeq 0.5 \%$ \\
$v_{\mathrm{S}}$ & $0.5 \%$ \\
$v_{S}$ & $0.3 \%$ \\
$v_{\mathrm{G}}$ & $0.2 \%$ \\
$v_{\mathrm{m}}\left(k_{\mathrm{x}}\right)$ & $1.1 \%{ }^{4}$ \\
\hline
\end{tabular}

${ }^{a} v_{m}\left(k_{x}\right)$ is a relative standard deviation of the mean for 9 degrees of freedom.

replicate evaluations of $v_{\mathrm{C}}$ and $v_{\mathrm{LS}}$ given in Fig. 3 or those for $v_{K}$ and $v_{\mathrm{S}}$ given in Table 3). If, of course, one had perfect knowledge of the underlying distributions from which the quantities were sampled, then the degrees of freedom would reveal these "uncertainties in the uncertainties." Examinations of the data sets for the various estimators like $v_{C}, v_{\mathrm{LS}}, v_{R}, v_{\mathrm{S}}$, or $v_{k \mathrm{x}}$ indicates that the relative uncertainty on these uncertainties are typically $50-200 \%$ (but can, as seen in some cases, substantially vary more widely). One must therefore recognize that such uncertainty estimators (or any that follow) are very crude, rough quantifications that reflect only general magnitudes or their orders. It is not an extreme statement to generalize that uncertainty estimators for complex experiments with many uncertainty components (even when the experiments are very carefully controlled and when the quantity of available data is vast) are usually very, very poorly known. One should not then place too much emphasis on any explicitly cited magnitude. To wit, for all intensive purposes two relative uncertainties quoted as, for example, $0.8 \%$ and $1.2 \%$ must be viewed as essentially equal with magnitude of about $1 \%$.

The generator, as presently evaluated, may be described in terms of the parameters [see eq. (1)] and conditions:

$f=0.625 \pm 0.005$

$A_{0}=1167 \pm 6 \mathrm{~Bq}$;

(as of $t_{0}=1200$ EST 9 September 1991);

$\lambda_{\mathrm{Ra}}=(1.186 \pm 0.005) \times 10^{-6} \mathrm{~d}^{-1}$;

$t_{\mathrm{d}}$ - variable from $t_{0}$, but presently $t_{\mathrm{d}}>1.7\left(\times 10^{3}\right) \mathrm{d}$;

$\lambda_{\mathrm{Rn}}=0.18130 \pm 0.00002 \mathrm{~d}^{-1}$

$t_{\mathrm{a}}=$ variable, but typically $t_{\mathrm{a}}>1 \mathrm{~d}$ for best precision;

$M=$ variable, with $M=m_{\mathrm{d}}+m_{\mathrm{D}}$ (best precision ob-

tained with $M \simeq 55 \mathrm{~g}$;

$m_{\mathrm{d}}-1.55 \pm 0.15 \mathrm{~g}$

$k_{\mathrm{x}}=0.784 \pm 0.009 \mathrm{~g}$.
The cited uncertainty (defined to be a standard uncertainty) on each of these. excepting that for $f$, were given in context earlier. That for $f$ is summarized in Table 6 . The uncertainty in the total ${ }^{222} \mathrm{Rn}$ activity $A$ (or activity concentration $K$ ) for a time $t_{2}$ at the end of an accumulation in a given dispensed aliquant from a typical generator run is summarized in Table 7. The assessment in Table 7 assumes that all masses (i.e., those required to obtain $m_{\mathrm{D}}$ as well as the dispensed aliquant $m_{j}$ ) were determined by careful gravimetric measurements. The additional uncertainty that results from use of the "mass per unit turn" conversion factor $k_{x}$ will be addressed subsequently. Th avoid uncertainty correlations for "double accounting" of uncertainty components such as for $A_{0}$ ). Table 7 utilizes only the reproducibility in $f$ (given by $v_{f} \simeq 0.3 \%$ instead of the entire combined standard uncertainty in $f$ of about $0.8 \%$ (Table 6 ). In addition, however, for any given run (generator operation), one must incorporate the attendant variabilities due to sampling precision (solution homogeneity) 's and generator precision $v_{\mathrm{G}}$ (the latter of which largely consists of $t_{\mathrm{M}}$ ).

It must be emphasized that the assessment of Table 7 is applicable only if $m_{\mathrm{D}}$ is obtained by mass measurements. If $M$ is estimated volumetrically, as in the original protocol [2], then the expanded uncertainties on $A$ and $K$ are likely to be several fold that given in Table 7 (i.e.. probably $>3 \%$ ). This is apparent from re-examination of the between-run reproducibility in Fig. 2 . In addition, if the dispensed aliquant $m_{j}$ is estimated by use of the $k_{x}$ "mass per unit turn" conversion factor, then the uncertainty component for $m_{j}$ in Table 7 must be increased to $\sqrt{10} \cdot t_{\mathrm{m}}\left(k_{\mathrm{x}}\right) \simeq 3.5 \%$ for any one dispensed aliquant. This

Table 6

Assessment of the uncertainty in the ${ }^{222}$ Rn emanation fraction $f$ for the Radon-In-Water Standard Generator. given in terms of the relative standard uncertainty (in \%) for each uncertainty component

Uncertainty component

Standard

uncertainty

Reproducibility $i_{f}$ in determining $f$ from

26 independent evaluations under many

variable conditions

Typical LS detection efficiency $\varepsilon_{\mathrm{s}} q$ used

to determine ${ }^{222} \mathrm{Rn}$ activity

${ }^{226} \mathrm{Ra}$ activity $A_{0}$ in source

${ }^{226} \mathrm{Ra}$ decay corrections, $\exp \left(-\lambda_{\mathbf{R a}} t_{\mathbf{d}}\right)$

${ }^{222} \mathrm{Rn}$ decay corrections for LS measurements.

$\exp \left(-i_{R \mathbf{n}} T_{i}\right)$

typical ${ }^{222} \mathrm{Rn}$ growth accumulation factors.

$\left[1-\exp \left(-\lambda_{\mathrm{R}_{\mathrm{n}}} t_{\mathrm{a}}\right)\right]$

Gravimetric measurement of any one

aliquant $m_{j}$ for LS measurements

Gravimetric determination of $M=m_{\mathrm{d}}+m_{\mathrm{D}}$

Combined standard uncertainty
$0.30 \%$

$0.30 \%$
$0.6 \%$

$0.47 \%$
$<0.001 \%$
$<0.0001 \%$
$<0.05 \%$

$0.05 \%$

$0.15 \%$
$0.84 \%$


Table 7

Assessment of the uncertainty in the ${ }^{222} \mathrm{Rn}$ activity concentration $K$ or total activity $A=K m_{j}$ in an aliquant $m_{j}$ dispensed from the Radon-In-Water Standard Generator, given in terms of the relative standard uncertainty (in \%) for each uncertainty component

\begin{tabular}{|c|c|}
\hline Uncertainty component & $\begin{array}{l}\text { Standard } \\
\text { uncertainty }\end{array}$ \\
\hline $\begin{array}{l}\text { Reproducibility } v_{f} \text { in determining } f \text { from } \\
26 \text { independent evaluations under many } \\
\text { variable conditions }\end{array}$ & $0.3 \%$ \\
\hline $\begin{array}{l}\text { Sampling precision } v_{\text {ss }} \text {, including solution } \\
\text { homogeneity (sample-to-sample variability) }\end{array}$ & $0.5 \%$ \\
\hline $\begin{array}{l}\text { Generator precision } v_{G} \text {, largely reflecting } \\
\text { the run-to-run reproducibility in determining } M\end{array}$ & $0.2 \%$ \\
\hline${ }^{226} \mathrm{Ra}$ activity $A_{0}$ in source & $0.47 \%$ \\
\hline${ }^{226} \mathrm{Ra}$ decay corrections, $\exp \left(-\hat{i}_{\mathrm{R}_{\mathrm{a}}} t_{\mathrm{d}}\right)$ & $<0.001 \%$ \\
\hline $\begin{array}{l}\text { Typical }{ }^{222} \mathrm{Rn} \text { growth accumulation factor. } \\
{\left[1-\exp \left(-i_{\mathrm{Rn}} t_{\mathrm{a}}\right)\right]}\end{array}$ & $<0.05 \%$ \\
\hline $\begin{array}{l}\text { Gravimetric measurement of any one } \\
\text { aliquant } m_{j}\end{array}$ & $0.05 \% \%^{\mathrm{a}}$ \\
\hline Gravimetric determination of $M=m_{\mathrm{d}}+m_{\mathrm{D}}$ & $0.2 \%$ \\
\hline Combined standard uncertainty & $0.80 \%$ \\
\hline Expanded uncertainty $(k=2)$ & $1.6 \%$ \\
\hline
\end{tabular}

${ }^{a}$ Required only for the uncertainty in the total ${ }^{222} \mathrm{Rn}$ activity $A=K m_{j}$ in a dispensed aliquant of mass $m_{j}$, not for the uncertainty in $K$.

uncertainty component would obviously dominate the uncertainty in $A=K m_{j}$. The $k_{\mathrm{x}}$-factor operating option was incorporated only for user convenience, and its use is not recommended except for applications that do not require a measurement accuracy of better than $5-10 \%$.

\section{Summary}

The performance efficacy and long-term stability of the NIST Radon-In-Water Standard Generator, previously developed and described [2], has been exhaustively evaluated over a period approaching 7 years.

Two revisions to the original operating protocol have been incorporated; viz., a minor one of increasing the number of syringe transfer mixings $n_{\mathrm{x}}$ from 2 to 3 (to improve solution homogeneity), and a more substantive change in the method used to determine the total ${ }^{222} \mathrm{Rn}$ solution volume (or mass $M$ ) by gravimetric means (to obtain substantially improved reproducibility in the ${ }^{222} \mathrm{Rn}$ concentration in a dispensed aliquant between generator runs). Evaluations of the ${ }^{222} \mathrm{Rn}$ emanation fraction $f$, the most critical generator parameter, have demonstrated that it has remained constant with a relative standard deviation of the mean of about $0.3 \%$, which indicates that the encapsulated ${ }^{226} \mathrm{Ra}$ source has not deleteriously aged or degraded with time.
The uncertainty (a relative combined standard uncertainty multiplied by $k=2$ ) associated with the ${ }^{222} \mathrm{Rn}$ activity concentration $K$ or total activity $A$ in any given dispensed aliquant from the generator (when the generator is operated by following the prescribed protocol) is typically $\pm 1.6 \%$.

The laboriously presented uncertainty treatments (with its attendant statistical-analysis model), as presented herein, may have pedagogic value for other researchers. The treatments clearly delineate the nature of nested uncertainties; which are associated with $n_{\mathrm{m}}$ multiple measurements on any given sample, with $n_{\mathrm{s}}$ multiple samples obtained from any given experimental trial (such as from particular runs of the generator as given here), and with multiples of such trials. Appropriate analyses of computed statistics (e.g., the standard deviations of directly measured quantities) can be used to derive underlying, inherent uncertainties in the measurement system itself (e.g., those for $v_{\mathrm{L}}, v_{\mathrm{s}}$, and $v_{\mathrm{G}}$ given herein). Continuous monitoring (and evaluations) of these system uncertainties can, and will prove to be of great benefit for routine monitoring of the performance of measurement systems and/or of assessing the quality of resultant mcasurement data obtained from those systems.

\section{Disclaimer}

Certain commercial equipment, instruments, or materials are identified in this paper to foster understanding. Such identification does not imply recommendation or endorsement by the National Institute of Standards and Technology, nor does it imply that the materials or equipment are the best available for the purpose.

\section{Acknowledgements}

The National Institute of Standards and Technology (NIST) is an agency of the Technology Administration, U.S. Department of Commerce. Dr. J.M.R. Hutchinson, of the NIST Radioactivity Group, assisted in many of the early experimental trials during the first year of these evaluations. He must be credited, and is also thanked, for his leadership in the development of the original radonin-water standard generator. Ms. Pamela A. Hodge and Mme. Dr. K.A. Maroufi-Collé are thanked for assistance in sorting and organizing the extensive data sets obtained over nearly 7 years. This journal's anonymous reviewer of this paper is heartily thanked for perceptively detecting and pointing out our original oversight in estimating the statistical "counting errors" for the radon-222 subseries. A co-operative agreement between NIST and the Food and Drug Administration (FDA) of the U.S. Department of Health and Human Services facilitated the participation of one of us (RK) during the initial evaluation period (1989-1991). 


\section{References}

[1] J.M.R. Hutchinson. P.A. Mullen, R. Collé, Nucl. Instr. and Meth. 223 (1984) 451

[2] J.M.R. Hutchinson, P.A. Mullen, R. Collé, Nucl. Instr. and Meth. A $247(1986) 385$.

[3] H.M. Pritchard, T.F. Gesell, Health Phys. 33 (1977) 577.

[4] J.E. Partridge. T.R. Horton, E.L. Sensintafar, A study of radon-222 released from water during typical household activities, U.S. Environmental Protection Agency Report EPA ORP/EERF-79-1, Montgomery. Alabama, 1979.

[5] T.R. Horton, Methods and Results of EPA's Study of Radon in Drinking Water, U.S. Environmental Protection Agency Report EPA 520/5-83-027. Montgomery, Alabama, 1993.

[6] W.A. Jester. P. Kotrappa, Trans. Amer. Nucl. Soc. 60 (1989) 88

[7] E.L. Whittaker, J.D. Akridge, J. Giovino, Two test procedures for radon in drinking water: interlaboratory collaborative study, U.S. Environmental Protection Agency Report EPA 600/2-87/082. Washington, DC, 1989.

[8] B. Parsa, T. Horton, Health Phys. 58 (1990) 209.

[9] E. Vitz, Health Phys. 60 (1991) 817.

[10] National Institute of Standards and Technology. Certificates for Standard Reference Material SRM 4965, 4966, and 4967, Radium-226 Radioactivity Standard, issued January 1992.

[11] R.Collé, P. Kotrappa, J.M.R. Hutchinson, J. Res. NIST $100(1995) 629$

[12] R. Collé. Radioact. and Radiochem. 6 (1) (1995) 16.

[13] J. Dean, National Physical Laboratory, Teddington, UK private communications (1994).

[14] International Organization for Standardization (ISO), Guide to the Expression of Uncertainty in Measurement, ISO, Geneva, Switzerland (1993). Jointly published by the International Bureau of Weights and Measures (BIPM). International Electrotechnical Commission
(IEC), International Federation of Clinical Chomistry (IFCC), ISO. International Union of Pure and Applicd Chemisty (IUPAC). International Union of Pure and Applied Physics (JUPAP), and the International Organization for Legal Metrology (OIML).

[15] B.N. Taylor, C.E. Kuyatt. Guidelines for Evaluating and Expressing the Uncertainty of NIST Measurement Results: 1994 Edition, National Institute of Standards and Technology (NIST) Technical 1297, U.S. Government Printing Office, Washington, D.C., 1994.

[16] Evaluated Nuclear Structure Data File (Oak Ridge Nuclear Data Project), Oak Ridge, TN. 1993.

[17] R. Colle, Radioact. Radiochem. 6 (1)(1995) 30.

[18] R. Collé. Radioact. Radiochem. 4 (1) (1993) 20.

[19] R. Collé, J.W.L. Thomas, J. Res. NIST 98 (1993) 653.

[20] R. Collé. Zhichao Lin, F.J. Schima. P.A. Hodge, J.W.L Thomas, J.M.R. Hutchinson, B.M. Coursey. J. Res. NIST $100(1995) 1$.

[21] B.E. Zimmerman. R. Colle, Standardization of $4.3 \mathrm{Ni}$ by $4 \pi \beta$ liquid scintillation spectrometry with ${ }^{3} \mathrm{H}$-standard efficiency tracing. J. Res. NIST 102 (1997) in press.

[22] R. Collé, Systematic effects of total cocktail mass (volume) and $\mathrm{H}_{2} \mathrm{O}$ fraction on $4 \pi \beta$ liquid scintillation spectrometry of ${ }^{3} \mathrm{H}$. Appl. Radiat. Isot. 48 (1997) in press.

[23] A. Grau Molonda. E. Garcia-Torano. Int. J. Appl. Radiat. Isot. 33 (1982) 249.

[24] B.M. Coursey, W.B. Mann, A. Grau-Molonda, E. GarciaTorano. Int. J. Appl. Radiat. Isot. 37 (1986) 403.

[25] B.M. Coursey. A. Grau Molonda. E. Garcia-Torano, J.M. Arcos. Trans. Amer. Nucl. Soc. 50 (1985) 13.

[26] R. Collé, J.M.R. Hutchinson. M.P. Unterweger, J. Res. NIST $95(1990) 155$.

[27] J.M.R. Hutchinson. J.Cessna, R. Collé, P.A. Hodge, Int. J. Appl. Radiat. Isot. 43 (1992) 175.

[28] G.W. Snedecor, W.G. Cochran, Statistical Methods, 6th ed.. Iowa State Univ. Press, Ames, IA, 1967.

[29] H.F. Lucas. J I.. D.A. Woodward, J. Appl. Phys. 35 (1964) 452. 